\title{
Abolition of aberrant neurogenesis ameliorates cognitive impairment after stroke in mice
}

\author{
María Isabel Cuartero, ,1,2 Juan de la Parra, 1,2 Alberto Pérez-Ruiz, ${ }^{1,2}$ Isabel Bravo-Ferrer, ${ }^{1,2}$ Violeta Durán-Laforet, ${ }^{1,2}$ \\ Alicia García-Culebras, ${ }^{1,2}$ Juan Manuel Carcía-Segura, ${ }^{2,3}$ Jagroop Dhaliwal, ${ }^{4}$ Paul W. Frankland, ${ }^{4}$ \\ Ignacio Lizasoain,, ${ }^{1,2}$ and María Ángeles Moro ${ }^{1,2}$ \\ 'Departamento de Farmacología y Toxicología, Facultad de Medicina, Universidad Complutense de Madrid (UCM), and Instituto de Investigación Hospital 12 de Octubre (i+12), Madrid, Spain. \\ ${ }^{2}$ Instituto Universitario de Investigación en Neuroquímica (IUIN), UCM, Madrid, Spain. ${ }^{3}$ Departamento de Bioquímica y Biología Molecular, Facultad de Ciencias Químicas, UCM, Madrid, Spain. \\ ${ }^{4}$ Program in Neuroscience \& Mental Health, Hospital for Sick Children, Toronto, Ontario, Canada.
}

\begin{abstract}
Poststroke cognitive impairment is considered one of the main complications during the chronic phase of ischemic stroke. In the adult brain, the hippocampus regulates both encoding and retrieval of new information through adult neurogenesis. Nevertheless, the lack of predictive models and studies based on the forgetting processes hinders the understanding of memory alterations after stroke. Our aim was to explore whether poststroke neurogenesis participates in the development of long-term memory impairment. Here, we show a hippocampal neurogenesis burst that persisted 1 month after stroke and that correlated with an impaired contextual and spatial memory performance. Furthermore, we demonstrate that the enhancement of hippocampal neurogenesis after stroke by physical activity or memantine treatment weakened existing memories. More importantly, stroke-induced newborn neurons promoted an aberrant hippocampal circuitry remodeling with differential features at ipsi- and contralesional levels. Strikingly, inhibition of stroke-induced hippocampal neurogenesis by

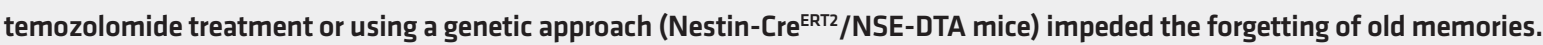
These results suggest that hippocampal neurogenesis modulation could be considered as a potential approach for treatment of poststroke cognitive impairment.
\end{abstract}

\section{Introduction}

Stroke is one of the leading causes of death and disability worldwide, affecting millions of lives every year. In the past few decades, advances in prevention and healthcare have progressively reduced stroke mortality (1). As a result, stroke can now be considered a chronically disabling disease, with many stroke survivors displaying a range of motor, cognitive, and psychiatric impairments. Importantly, whereas motor impairment may ameliorate during the chronic phase of stroke, cognitive deficits tend to worsen (2, $3)$. In fact, more than one-third of patients may develop cognitive impairment or even dementia later after stroke (4-6). Despite this high prevalence, mechanisms underlying poststroke cognitive impairment and dementia remain unclear $(2,4,7)$.

The hippocampus is one of the main adult brain regions implicated in cognitive functions. Through adult neurogenesis, newborn neurons are continually added to the hippocampal circuits, contributing to the encoding of new hippocampus-dependent memories (8). Nevertheless, since neuronal integration produces remodeling of preexisting hippocampal circuitry, increasing neurogenesis also may promote the destabilization and even the

Authorship note: MIC, JDLP, and ARP have contributed equally to this work. Conflict of interest: The authors have declared that no conflict of interest exists. Copyright: () 2019 American Society for Clinical Investigation Submitted: February 7, 2018; Accepted: January 17, 2019. Reference information: / Clin Invest. 2019;129(4):1536-1550. https://doi.org/10.1172/JCl120412. clearance of previous stored memories (9). Indeed, alterations in neurogenesis and neuronal integration have been proposed as contributing to cognitive impairment (10-12) in different pathological scenarios, such as epilepsy, schizophrenia, and neurodegenerative diseases (for review, see refs. 8, 13).

Of note, stroke is known to trigger a strong neurogenic burst in the hippocampus (for review, see refs. 14, 15). Although subgranular zone (SGZ) neurogenesis could promote cognitive function recovery after cerebral ischemia $(16,17)$, stroke also modifies the morphology of newborn granule cells $(18,19)$, concomitant to hippocampus-dependent memory deficits, suggesting a role of hippocampal neurogenesis in poststroke cognitive impairment. Furthermore, higher levels of hippocampal neurogenesis promote forgetting of old memories, which are otherwise strengthened by reducing neurogenesis $(9,20)$. We therefore explored hippocampus-dependent cognitive function as well as hippocampal neurogenesis and circuitry remodeling response after stroke and examined neurogenesis modulation to determine whether it might ameliorate hippocampus-dependent cognitive function.

\section{Results}

Cortical ischemia induces long-term hippocampus-dependent cognitive impairment. We first evaluated how permanent cerebral ischemia affects the formation of enduring memories using contextual fear conditioning (CFC) (Figure 1, A-C). Seven days after surgery, sham-operated and ischemic mice were placed in a chamber (context); after a familiarization phase, several electrical foot shocks 
A

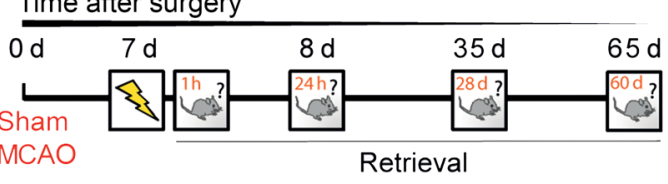

B

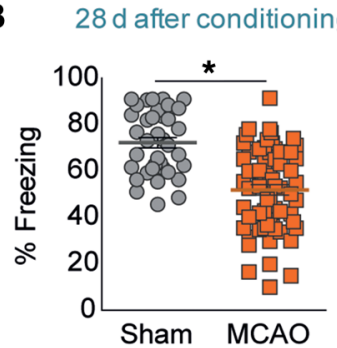

G

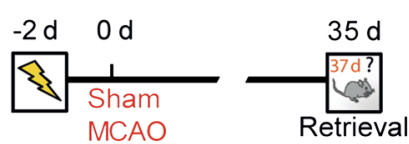

C
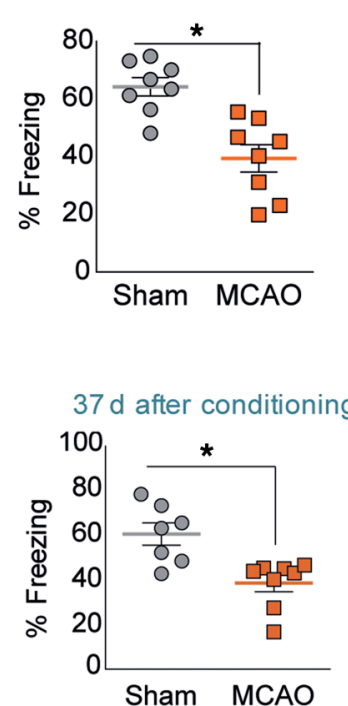

D

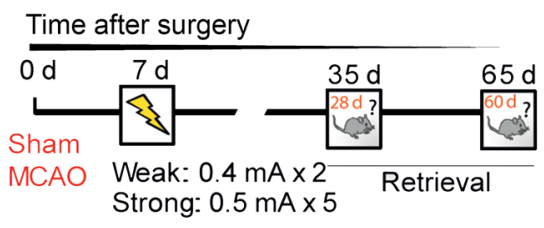

E
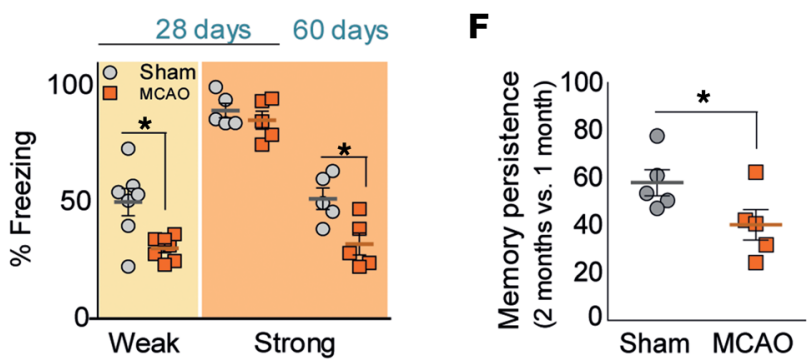

H

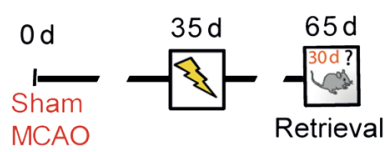

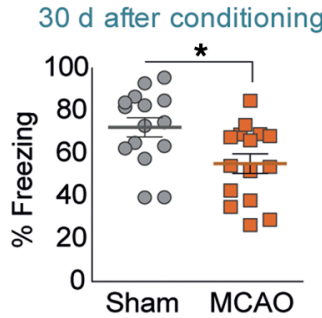

Figure 1. Cortical stroke impairs long-term memory in mice. (A) Experimental design for panels $\mathbf{B}$ and $\mathbf{C}$. Sham-operated and MCAO mice were subjected to CFC $(0.6 \mathrm{~mA} \times 3)$ and tested 28 and 60 days after training. (B and $\mathbf{C})$ Remote memory retention after cerebral ischemia calculated as the percentage of freezing response 28 days (B, $P<0.05$ vs. sham operated; sham operated, $n=37 ;$ MCAO, $n=63$ ) and 60 days (C, ${ }^{*} P<0.05$ vs. sham-operated group; sham operated, $n=8$; MCAO, $n=8$ ) after foot shocks. (D) Experimental design for panels $\mathbf{E}$ and $\mathbf{F}$. (E) Percentage of freezing in control and MCAO groups after a weak ( $0.4 \mathrm{~mA} \times 2$; left columns; light orange panel; ${ }^{*} P<0.05$ vs. sham-operated group; sham operated, $\left.n=7 ; \mathrm{MCAO}, n=7\right)$ or a strong fear-conditioning paradigm ( $0.8 \mathrm{~mA} \times 5$; right columns; dark orange panel; $P>0.05$ vs. sham-operated group; sham operated, $n=5 ;$ MCAO, $n=5$ ). Retention for both types of conditioning was performed 28 days after training and 60 days after conditioning for the strong one. (F) Memory persistence at 2 months after strong fear-conditioning paradigm. Data are represented as percentage of freezing at 2 months versus that observed at 1 month $\left({ }^{*} P<0.05\right.$ vs. sham-operated group; sham operated, $n=5$; MCAO, $n=5)$. (G and $\mathbf{H})$ Freezing response after conditioning $(0.6 \mathrm{~mA} \times 3)$ performed 48 hours before surgery $\left(\mathbf{G},{ }^{*} P<0.05\right.$ vs. sham operated; sham operated, $n=9$; MCAO, $n=7$ ) or 30 days after MCAO (H, ${ }^{*} P<0.05$ vs. sham operated; sham operated, $n=15 ;$ MCAO, $\left.n=15\right)$, respectively. Data are represented as mean \pm SEM. Data were compared using nonparametric 2-tailed Mann-Whitney $U$ test.

were presented, and recent and remote memory ( 24 hours, 28 days, or 60 days after conditioning) were calculated by measuring the freezing response. Sham-operated and ischemic mice behaved similarly during the training phase, displaying comparable exploratory activity and foot-shock reactivity (Supplemental Figure 1, A and $\mathrm{B} ; P>0.05$; supplemental material available online with this article; https://doi.org/10.1172/JCI120412DS1). In addition, the formation of new memories was not affected by middle cerebral artery occlusion (MCAO), since both sham-operated and MCAO mice displayed similar conditioned response 1 hour after training (Supplemental Figure 1C; $P>0.05$ ).

During the retention session, although sham-operated and MCAO groups did not show any differences when tested 24 hours after training (Supplemental Figure 1D; $P>0.05$ ), ischemic mice displayed decreased retrieval compared with that of the sham-operated group when remote memory was evaluated 28 days later (Figure 1B; $P<0.05$ vs. sham-operated). Importantly, this effect persisted at least 60 days after surgery (Figure 1C; $P<0.05$ vs. sham operated).

The degree of memory retention could be modulated by modifying initial memory strength by using a weak or a strong contextual fear training paradigm (Figure 1, D-F): while ischemic mice displayed less freezing behavior than the sham-operated group 28 days after foot-shock presentation when using a weak fear conditioning protocol (Figure 1E; $P<0.05$ vs. sham operated), differences between sham-operated and MCAO mice were abolished by a strong fear protocol (Figure 1E; $P>0.05$ vs. sham operated). Importantly, even with the latter, ischemic mice presented a low ratio of memory persistence when evaluated 60 days after training (Figure 1F; $P<0.05$ vs. sham operated), probably indicating a progressive cognitive decline that mimics that observed in stroke patients (3). We next asked whether the timing of new memory acquisition affects long-term memory retention and whether the long-term memory deficits dissipate with time. Of note, when conditioning was performed either 48 hours before surgery (Figure $1 \mathrm{G} ; P<0.05$ vs. sham operated) or 35 days after MCAO (Figure $1 \mathrm{H}$; $P<0.05$ vs. sham operated), similar results were observed, indicating that cerebral ischemia impairs the recall of remote memories independently of the time at which those memories were formed (i.e., before, early after, or even later after stroke).

Memory deficits after stroke are considered to be size and location dependent (for review, see ref. 21). In our model, in which lesions are restricted just to the cortex and the initial and final 
A
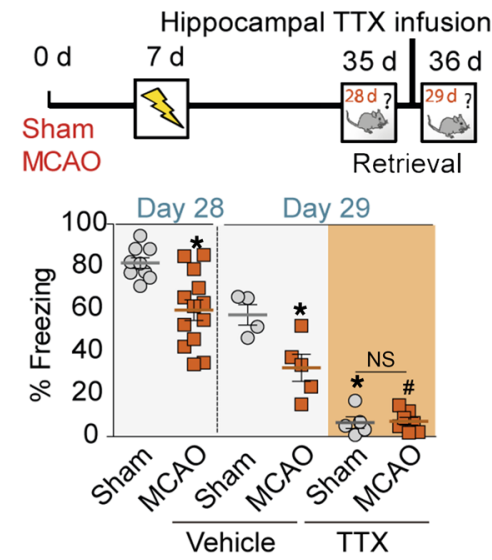

C

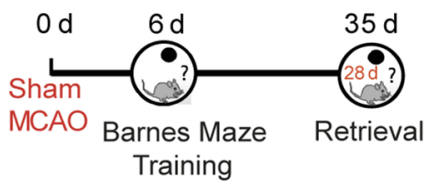

D

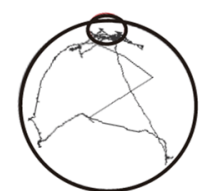

Sham

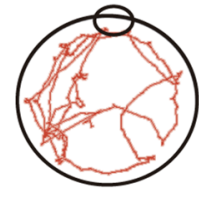

MCAO
B

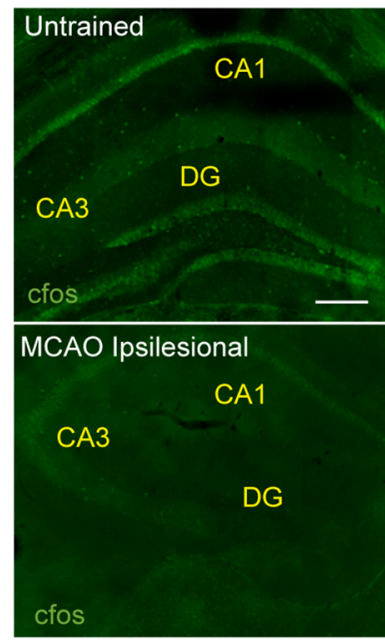

E

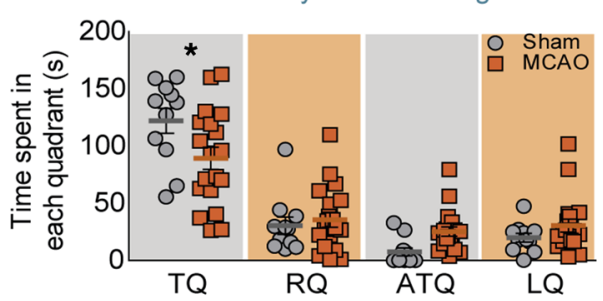

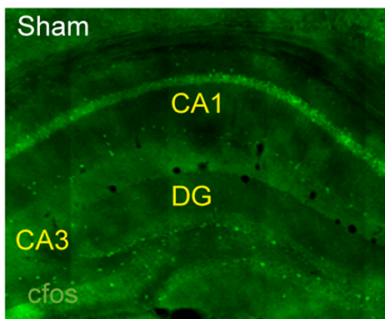

MCAO Contralesional

CA1
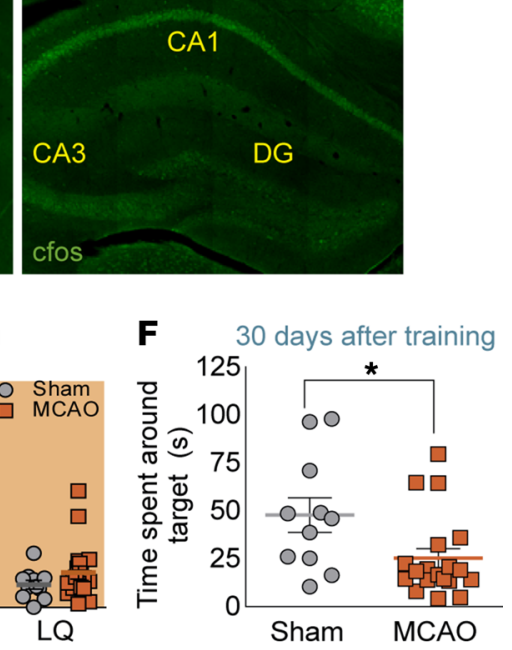

Figure 2. Long-term memory deficits after MCAO are hippocampus dependent. (A) Design for hippocampus inactivation with TTX before retrieval of fear memory. Sham-operated and MCAO mice were subjected to CFC $(0.6 \mathrm{~mA} \times 3) 7$ days after surgery and tested 28 days after training (* $P<0.05$ vs. sham operated; sham operated, $n=9$; MCAO, $n=13$ ). One day later, sham-operated and MCAO mice, allocated in 2 different groups, were bilaterally infused in the dorsal hippocampus with either vehicle or TTX. Three hours later, fear memory was evaluated as percentage of freezing (sham-operated vehicle, $n=$ 4; MCAO vehicle, $n=5$; sham-operated TTX, $n=5$; MCAO TTX, $n=8$; ${ }^{*} P<0.05$ vs. sham-operated vehicle; ${ }^{*} P<0.05$ vs. MCAO vehicle). (B) Representative images of c-Fos staining (green) in the hippocampus of untrained, sham-operated, and ischemic mice 90 minutes after a 10 -minute retrieval session, 28 days after conditioning. Scale bar: $200 \mu \mathrm{m}$. (C-F) Barnes maze testing. (C) Experimental design. (D) Representative traces of the paths traveled during retrieval by mice in the Barnes maze obtained by EthoWatcher software. Time spent by sham-operated and MCAO mice around each quadrant (E) or around the target hole (F) in the Barnes maze platform 30 days after training. For $\mathbf{E}$, 2-way ANOVA showed a significant interaction between quadrants and surgery $\left(F_{(3,116)}=3.50 ; P=0.0178\right)$ (Bonferroni's post hoc: ${ }^{*} P<0.05$ vs. sham-operated TQ; sham operated, $\left.n=12 ; M C A O ; n=19\right)$. RQ, right quadrant; ATQ, antitarget quadrant; LQ, left quadrant. For $\mathbf{F},{ }^{*} P<0.05$ vs. sham-operated group. Data are represented as mean \pm SEM. Data were compared by using nonparametric 2-tailed Mann-Whitney $U$ tests (A and F) or nonparametric 2-way ANOVA followed by Bonferroni's post hoc testing (E).

lesions are directly correlated (Supplemental Figure $2 \mathrm{~A}, P<0.05$ ), we did not detect any association between damage and degree of cognitive function, determined as freezing response (Supplemental Figure 2B; $P>0.05)$. Likewise, similar infarct volumes were observed along the anteroposterior axis (Supplemental Figure 2C), with an exclusive cortical affectation, showing that memory deficits were not causally related to infarct size or location.

Enduring contextual fear-conditioning memories have been ascribed to the hippocampus (22). To determine whether cognitive impairment was due to the specific involvement of hippocampus, we examined the ability of retrieval of remote memories after hippocampal inactivation in both sham-operated and ischemic mice (Figure 2A). First, 7 days after the surgery, mice were trained in CFC and tested 28 days after. Twenty-four hours later, mice received a bilateral infusion of vehicle or tetrodotoxin (TTX) in the dorsal hippocampus, and the freezing response was reassessed 3 hours later. TTX administration in both sham-operated and MCAO groups impaired retrieval compared with that of the vehicle-treated group, indicating that memory recall after fear conditioning in both groups was allocated to the hippocampus (Figure $2 \mathrm{~A} ; P<0.05$ vs. sham operated).
Accordingly, an altered pattern of hippocampal c-Fos expression was observed in ischemic mice after the retrieval of long-term CFC (Figure 2B). While 90 minutes after the retrieval session, conditioned sham-operated mice displayed an increase in the percentage of $\mathrm{c}-\mathrm{Fos}^{+}$ cells in different hippocampal subfields, CA1, CA3, and dentate gyrus (DG), relative to the unconditioned sham-operated group (mice that were exposed to the context during the retrieval session, but did not receive any foot shock 28 days before), the population of hippocampal neurons activated by context reexposure in ischemic mice was smaller in both ipsi- and the contralesional hippocampus compared with that of the sham-operated group (Figure 2B; c-Fos ${ }^{+}$cells vs. unconditioned mice: sham operated, $3.79 \pm 0.43$; MCAO ipsilesional, $1.59 \pm 0.29 ;$ MCAO contralesional, $1.51 \pm 0.36, n=6-7$ mice/group; sham operated vs. MCAO ipsilesional, $P=0.012$; sham operated vs. MCAO contralesional, $P=0.033$ ), reinforcing the implication of the hippocampus in MCAO-induced retrieval impairment.

Finally, we explored whether ischemia could also affect other types of hippocampus-dependent memory, such as spatial memory (Figure 2, C-F). Seven days after surgery, mice were trained for 6 consecutive days ( 3 rounds/d) in the Barnes maze platform, 
A
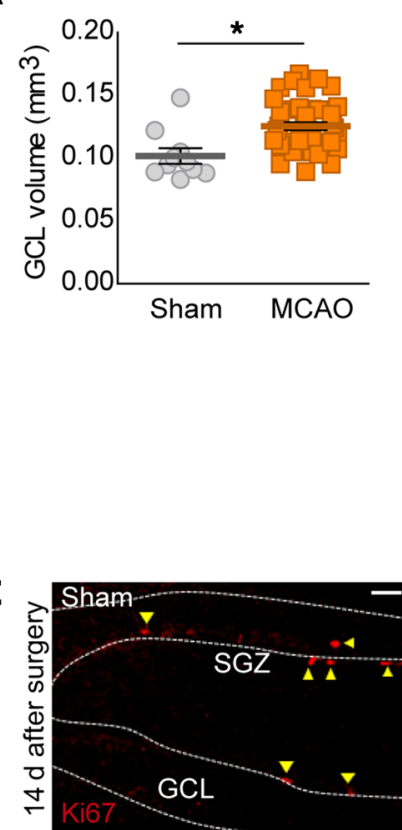

G

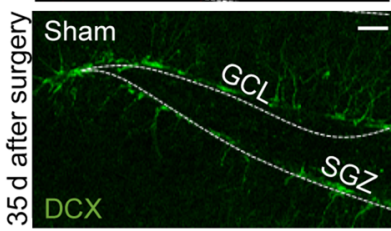

I

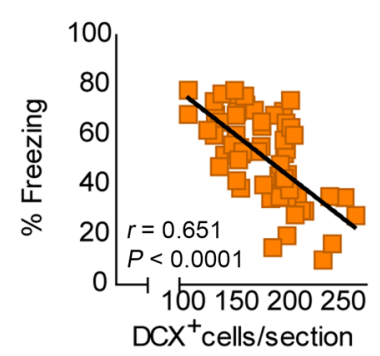

B
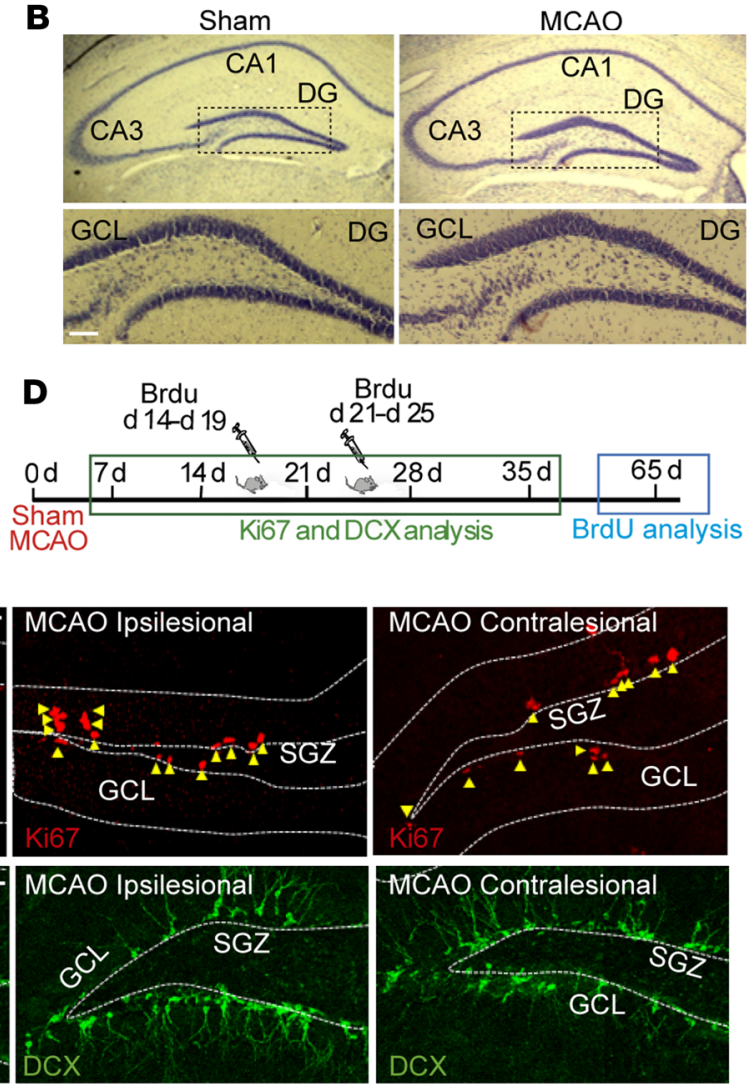

J
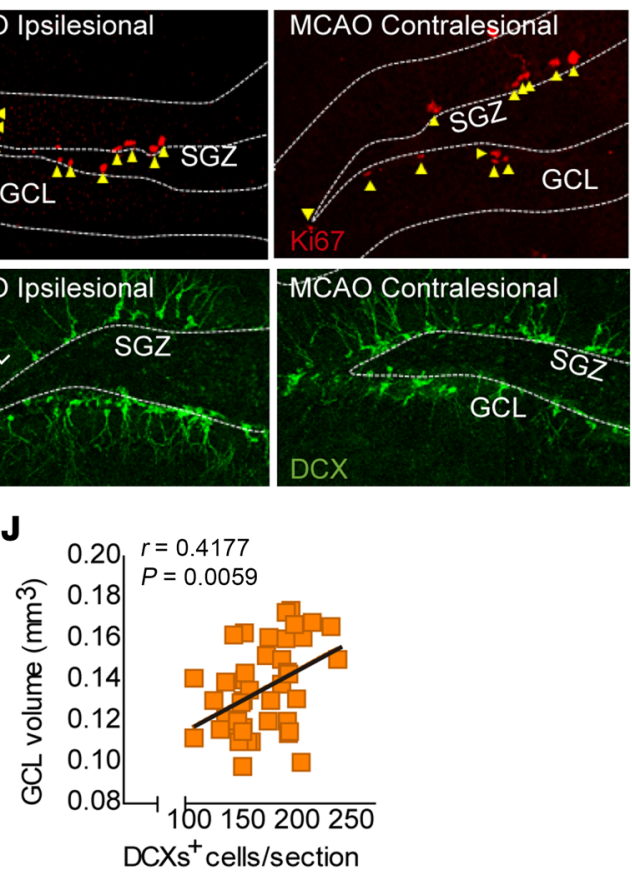

C
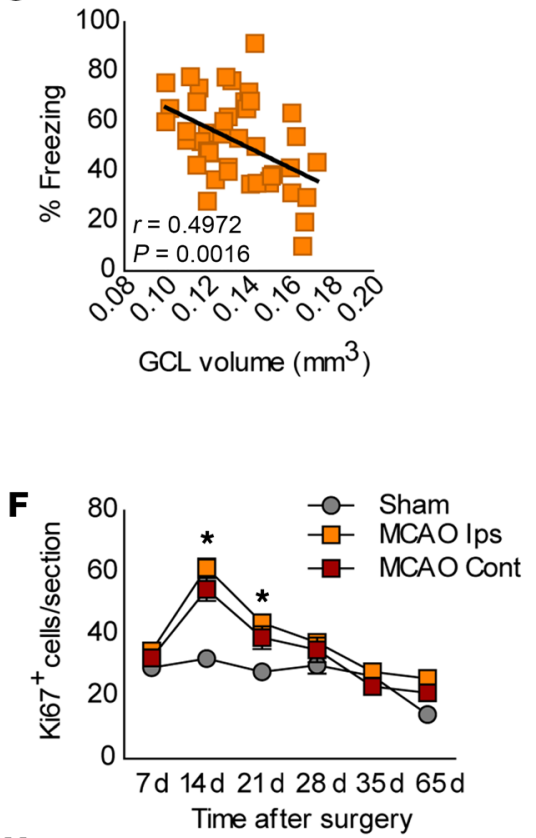

H

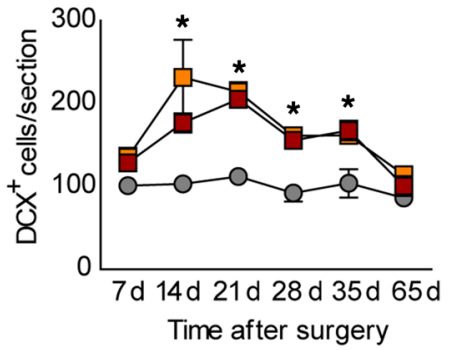

Figure 3. Hippocampal neurogenesis positively correlates with memory impairment. (A and B) Cavalieri estimation of GCL volume in Nissl-stained sections from sham-operated and MCAO mice 35 days after surgery ( ${ }^{*} P<0.05$ vs. sham operated; sham operated, $n=10 ;$ MCA0, $n=40$ ). (B) Representative images. Scale bar: $100 \mu \mathrm{m}$. (C) Negative relationship between GCL volume and freezing response in the ischemic group. Linear regression analysis is displayed (MCAO, $n=44$; Spearman's, $r=-0.4972, P=0.0016)$. (D) Experimental design for cell quantification. (E-H) Quantification of proliferative Ki67 ${ }^{+}$cells (F) and DCX+ immature neurons (H) in sham-operated and MCAO mice (ipsi- and contralesional DC). For $\mathbf{F}$, a significant interaction between time after surgery and ischemia was found for $\mathrm{Ki}^{+} 7^{+}\left(\mathrm{F}_{(10,59)}=3.5 ; P=0.0010\right.$; Bonferroni's post hoc: ${ }^{*} P<0.05$ vs. sham operated; sham operated, $n=4-5$; MCAO ipsilesional, $n=4-5$; MCAO contralesional, $n=3-5)$. For $\mathbf{H}$, 2-way ANOVA showed significant differences for ischemia $\left(F_{(2.57)}=27.90 ; P<0.0001\right)$ and for time after surgery $\left(F_{(5.57)}=\right.$ 5.57; $P<0.0001$ ) (Bonferroni's post hoc: ${ }^{*} P<0.05$ vs. sham operated; sham operated, $n=3-5 ;$ MCAO ipsilesional, $n=3-5 ;$ MCAO contralesional, $n=3-5$ ). (E and G) Representative images of $\mathrm{Ki}^{+}$(red) and DCX+ cells (green). Scale bar: $30 \mu \mathrm{m}$. (I and J) Spearman's correlation between ipsilesional DCX+ cells and percentage of freezing 35 days after surgery (I, MCAO, $n=66$; Spearman's, $r=-0.651, P<0.0001$ ) or between DCX+ cells and GCL volume in ischemic mice (MCA0, $n=40$; Spearman's, $r=0.4177, P=0.0059)$. Data are represented as mean \pm SEM. Data were compared by using nonparametric 2 -tailed Mann-Whitney $U$ tests (A) and nonparametric 2-way ANOVA followed by Bonferroni's post hoc test. (E and G) Correlation analysis was assessed by Spearman's (C, I, and J).

and 28 days later, a probe trial was conducted without the escape box to measure spatial memory retention. Both sham-operated and ischemic mice displayed similar speed and distance travelled and learned similarly to locate the escape hole with the hidden box during the course of the training period, as indicated by a progressive reduction in the time needed to reach the target hole (Supplemental Figure 3, A-D). However, confirming our previous results, ischemic mice displayed a decrease in spatial memory retention 28 days after training compared with the sham-operated group, when estimated either as the time spent in the target quadrant (TQ) (Figure 2E; $P<0.05$ vs. sham-operated TQ) or as the time spent around the target hole (Figure $2 \mathrm{~F} ; P<0.05$ vs. sham operated). All these data suggest that cortical stroke in mice promotes long-term hippocampus-dependent cognitive impairment.

Stroke-induced neurogenesis in the SGZ positively correlates with memory impairment after cerebral ischemia. Cortical focal ischemia produces a long-term memory impairment that seems to be due to hippocampal affectation. Although no changes were found in overall 
A

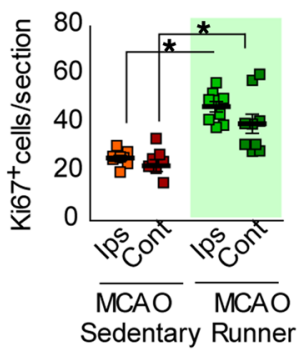

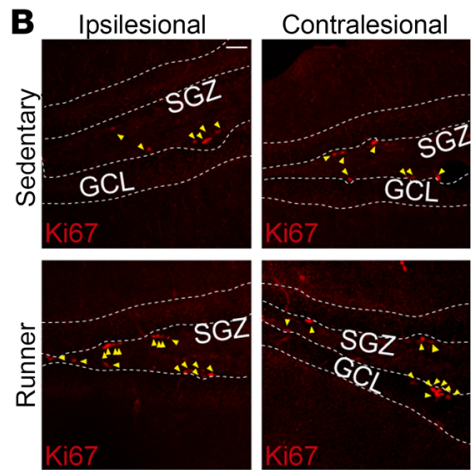

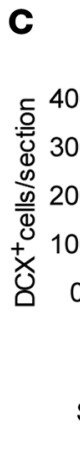

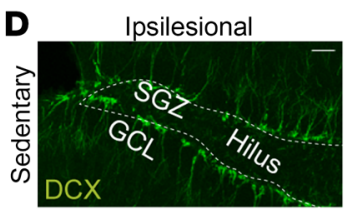
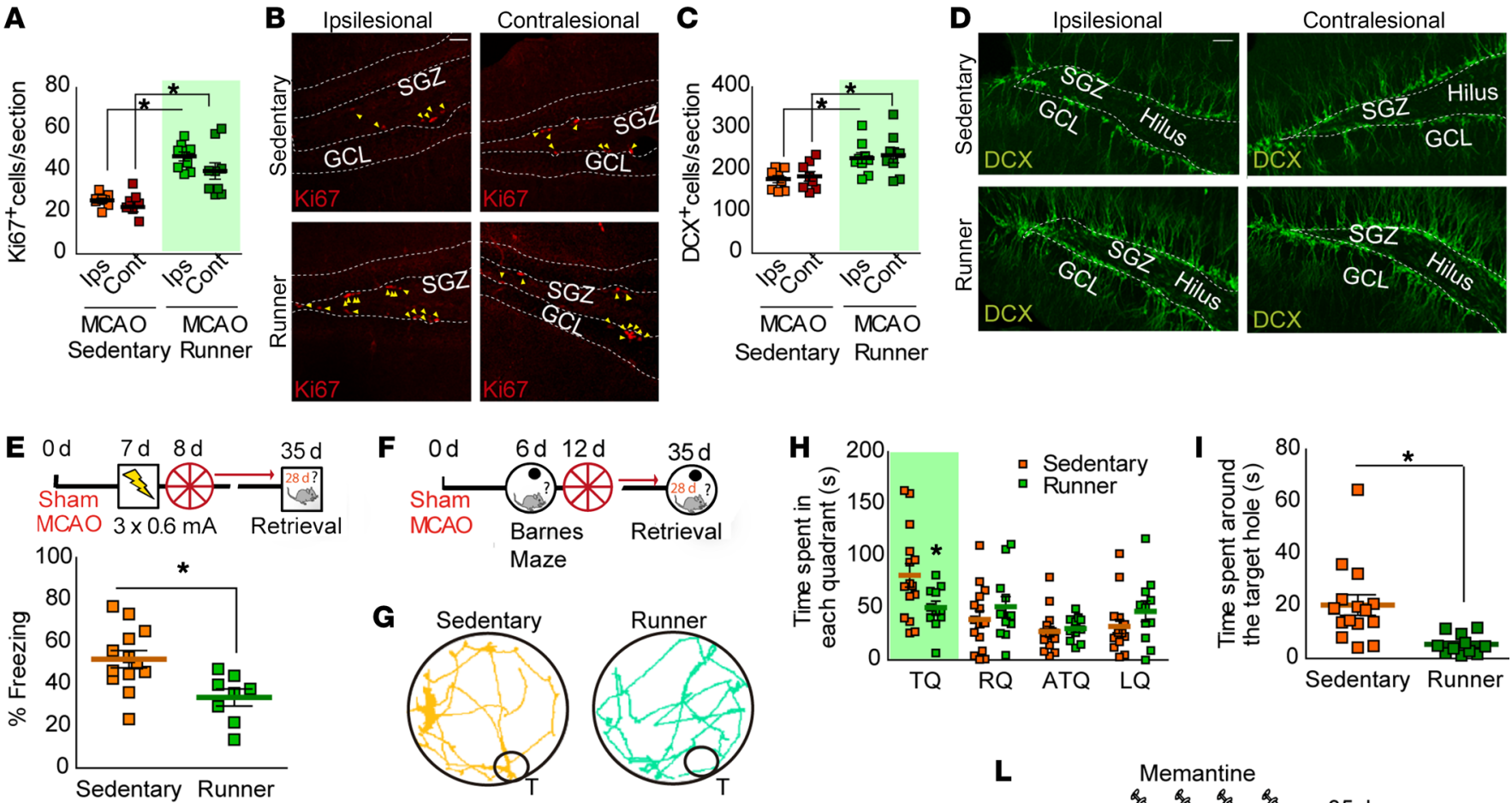

G
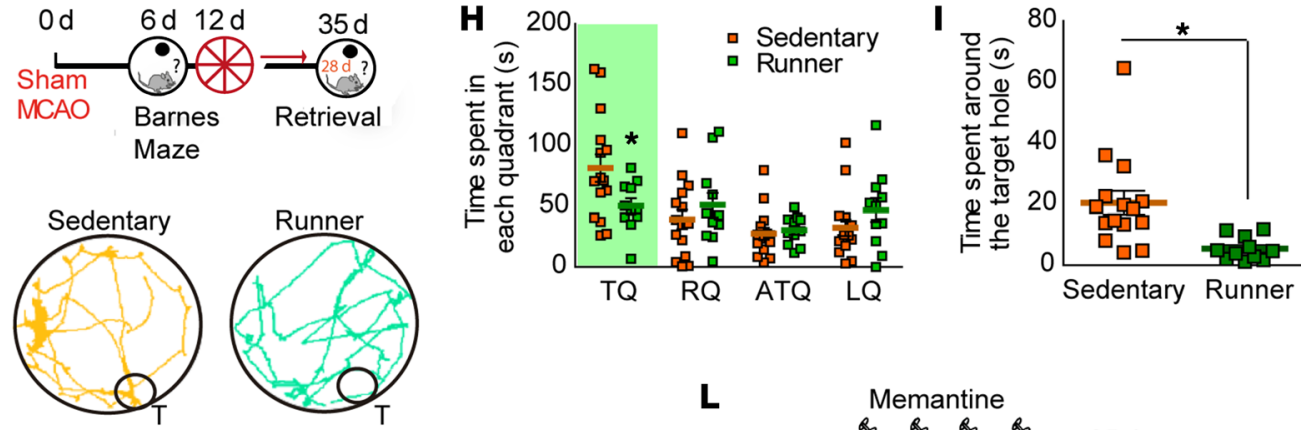

$J$

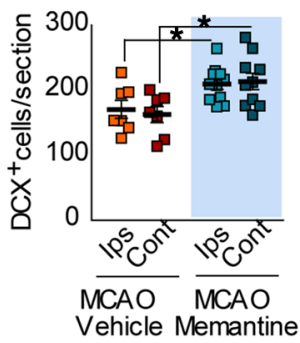

$\mathbf{K}$

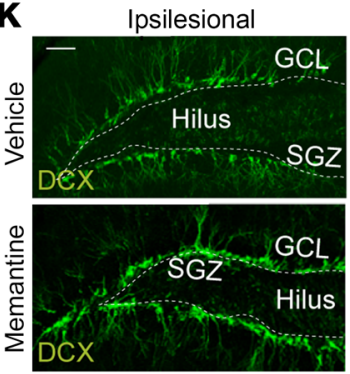

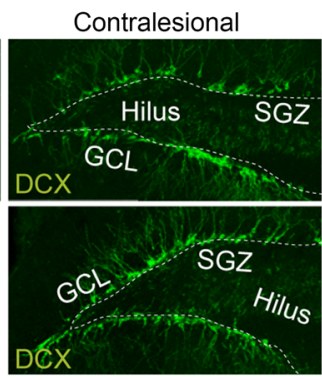

$\mathbf{L}$

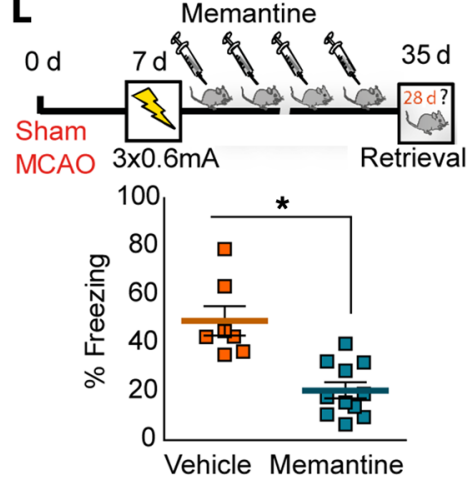

Figure 4. Increasing poststroke neurogenesis enhances cognitive impairment. (A-D) Quantification of Ki67+ (A) and DCX+ cells (C) 35 days after surgery in sedentary and runner MCAO mice. (B, D) Representative images. Scale bars: $30 \mu \mathrm{m}$. Two-way ANOVA analysis showed significant effect of running in number of $\mathrm{Ki67}^{+}(\mathbf{A})\left(\mathrm{F}_{(1,30)}=50.09 ; P<0.0001\right)$ and DCX+ cells $(\mathbf{C})\left(F_{(1,29)}=14.05 ; P=0.0008\right)$ (Bonferroni's post hoc: ${ }^{*} P<0.05$ vs. MCAO sedentary; $M C A 0$ sedentary, $n=8$; MCAO runner, $n=9$ ). (E) MCAO mice remained sedentary or ran after CFC and were tested 28 days later $\left({ }^{*} P<0.05\right.$ vs. MCAO sedentary; MCAO sedentary, $n=13$; MCAO runner, $n=8)$. ( $\mathbf{F}-\mathbf{I})$ Barnes maze testing. $(\mathbf{F})$ Experimental design. (C) Representative traces. Time spent by MCAO mice around each quadrant (H) or the target hole (I). For $\mathbf{H}, 2$-way ANOVA demonstrated significant interaction between different quadrants and running $\left(\mathrm{F}_{(3.96)}=3.25 ; P=0.0251 ;{ }^{*} P<0.05\right.$ vs. MCAO sedentary TQ; Bonferroni's post test). For I, ${ }^{*} P<0.05$ vs. MCAO sedentary; MCAO sedentary, $n=15$; MCAO runner, $n=11$. (J-L) MCAO mice treated with MEM showed a reduced freezing response $(\mathbf{L})\left({ }^{*} P<0.05\right.$ vs. MCAO vehicle; MCAO vehicle, $n=7 ;$ MCAO MEM, $\left.n=11\right)$ and an increased the number of DCX ${ }^{+}$cells (J and K). (J) Two-way ANOVA demonstrated significant effect of MEM in DCX+cells $\left(F_{(1,33)}=28.85 ; P<0.0001\right)$ (Bonferroni's post hoc: ${ }^{*} P<0.05$ vs. MCAO vehicle; MCAO vehicle, $n=7$; MCAO MEM, $n=10$ ). Representative images (K). Scale bar: $30 \mu \mathrm{m}$. Data are represented as mean \pm SEM. Data were compared by using nonparametric 2-tailed Mann-Whitney $U$ tests (E, I, and $\mathbf{L}$ ) and nonparametric 2-way ANOVA followed by Bonferroni's post hoc testing (A, C, $\mathbf{H}$ and J).

hippocampal morphology or volume either 1 or 2 months after ischemia using Nissl staining or MRI, respectively (Supplemental Figure 4, A-D; $P>0.05$ ), MCAO mice presented a significant increase in DG granular cell layer (GCL) volume compared with the sham-operated group 35 days after surgery (Figure 3, A and B; $P<0.05$ vs. sham operated). Interestingly, a negative relationship between GCL volume and freezing response was found in the ischemic group (Figure $3 C ; r=-0.4972, P=0.0016)$, indicating that animals with higher volumes displayed more severe cognitive deficits at the time studied.

In the SGZ of the DG, new granular neurons are generated throughout life. These newborn neurons integrate into hippocampal circuits that play a role in memory storage (23-25). Acute brain inju- ry stimulates hippocampal neurogenesis in different experimental models, but this response might have detrimental consequences and promote hippocampal malfunction, as observed in different pathologies, such as epilepsy or schizophrenia (8). Increased SGZ neurogenesis has also been found in different cortical stroke models $(26,27)$. Since perturbations in neurogenesis may alter GCL/DG size, we reasoned that poststroke neurogenesis could be involved in memory impairment observed after stroke. In our MCAO model (Figure 3D), an almost 2-fold increase in the number of proliferating $\mathrm{Ki}^{+} \mathrm{7}^{+}$cells and immature doublecortin ${ }^{+}\left(\mathrm{DCX}^{+}\right)$neurons was found in both ipsi- and contralesional SGZ14 days after injury, and this persisted at least 21 and 35 days after ischemia, respectively (Figure 3, 
A

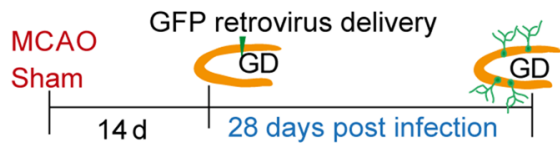

B
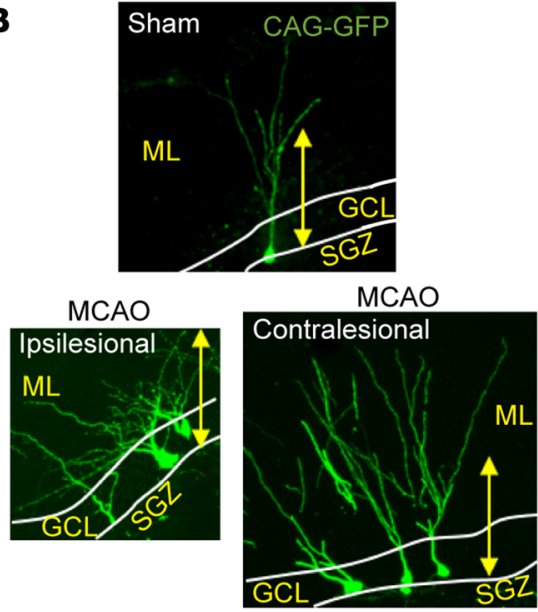

E

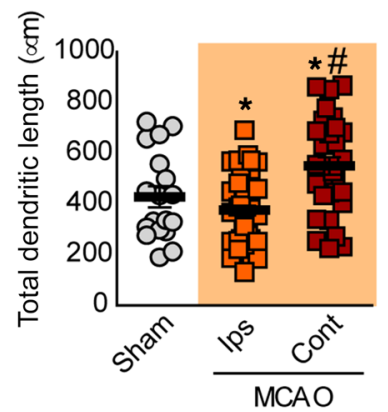

$\mathbf{F}$

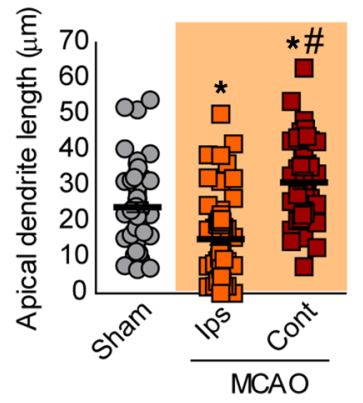

C

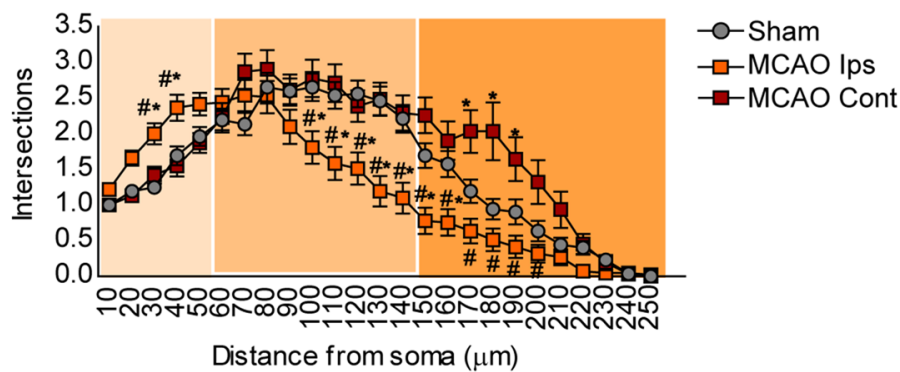

D

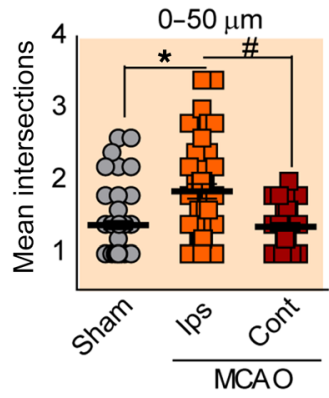

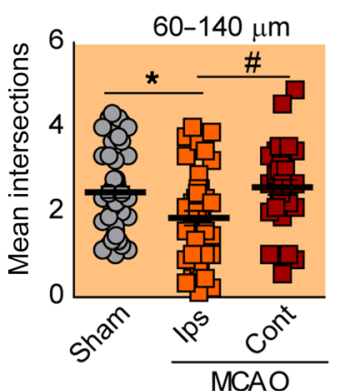

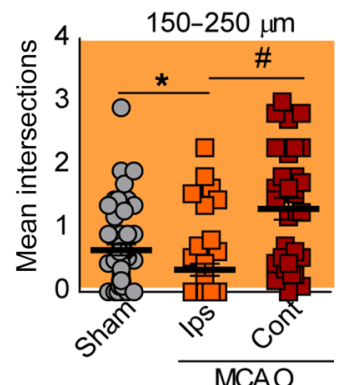

G

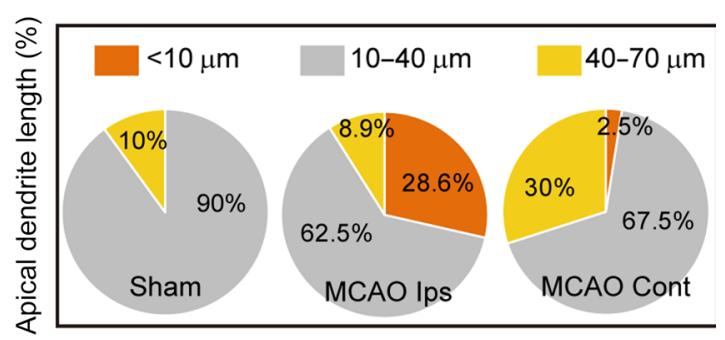

Figure 5. Altered features of newborn neurons induced by stroke. (A) Experimental design for GFP retroviral labeling of newborn neurons. (B) Representative images of GFP newborn neurons of each group. Yellow arrow, $112 \mu \mathrm{m}$. (C) Sholl analysis of GFP newborn neurons in sham-operated and MCAO groups, 28 days after infection. Two-way ANOVA demonstrates a significant interaction between distance and experimental group in the number of intersections $\left(\mathrm{F}_{(48,3022)}=5.18 ; P<0.0001\right.$; Bonferroni's post hoc: ${ }^{*} P<0.05$ vs. sham operated; ${ }^{*} P<0.05$ vs. MCAO ipsilesional; sham operated, $n=52$ neurons/4 mice; MCAO ipsilesional, $n=41$ neurons/4 mice; MCAO contralesional, $n=32$ neurons/3 mice). (D) Mean average intersections of GFP neurons at different intervals from soma. ( ${ }^{*} P<0.05$ vs. sham operated; ${ }^{~} P<0.05$ vs. MCAO ipsilesional; sham operated, $n=52$ neurons/4 mice; MCAO ipsilesional, $n=41$ neurons $/ 4$ mice; MCAO contralesional, $n=32$ neurons $/ 3$ mice). (E and $\mathbf{F})$ Quantification of total dendritic length $\left(\mathbf{E},{ }^{*} P<0.05\right.$ vs. sham operated; ${ }^{\#} P<0.05$ vs. MCAO ipsilesional, respectively; sham operated, $n=18$ neurons $/ 4$ mice; MCAO ipsilesional, $n=45$ neurons $/ 4$ mice; MCAO contralesional, $n=29$ neurons $/ 3$ mice), and apical dendritic length $\left(\mathbf{F},{ }^{*} P<0.05\right.$ vs. sham operated; ${ }^{*} P<0.05$ MCAO vs. ipsilesional; sham operated, $n=61$ neurons $/ 4$ mice; MCAO ipsilesional, $n=60$ neurons/4 mice; MCAO contralesional, $n=40$ neurons/3 mice) in sham-operated and both ipsi- and contralesional sides of MCAO group. (G) Pie charts display percentage of GFP+ neurons in each group showing apical dendrite lengths of less than $10 \mu \mathrm{m}, 10-40 \mu \mathrm{m}$, and more than $40 \mu \mathrm{m}$ (sham operated, $n=61$ neurons $/ 4$ mice; MCAO ipsilesional $n=60$ neurons $/ 4$ mice; MCAO contralesional, $n=40$ neurons $/ 3$ mice). Data are represented as mean \pm SEM. Data were compared by using nonparametric 2-tailed Mann-Whitney $U$ tests (D-F) and nonparametric 2-way ANOVA followed by Bonferroni's post hoc testing (C).

$\mathrm{E}-\mathrm{H} ; \mathrm{P}<0.05$ vs. sham operated). Importantly, we found an inverse correlation between the numbers of $\mathrm{DCX}^{+}$cells and the freezing response, indicating that increased neurogenesis after cerebral ischemia leads to more severe cognitive deficits (Figure 3I; Spearman's, $r=-0.651, P<0.0001)$. Accordingly, a positive correlation was found between GCL volume and $\mathrm{DCX}^{+}$cell number in the SGZ of ischemic mice (Figure 3J; Spearman's, $r=0.4177, P=0.0059$ ). We did not detect any differences between sham-operated and MCAO groups in the number of cleaved-caspase- $3^{+}$cells (data not shown), but we observed an approximately 1.5 -fold increase in the number of new neurons $\left(\mathrm{BrdU}^{+} / \mathrm{NeuN}^{+}\right.$cells) in both ipsi- and contralesional hippocampus 65 days after ischemia (Supplemental Figure 5, A-D; $P<0.05$ vs. sham operated; sham operated, 503.61 \pm 33.40 cells; $\mathrm{MCAO}$ ipsilesional, $766.52 \pm 38.33$ cells; MCAO contralesional, $723.34 \pm 34.57$ cells), indicating that the increase in $\mathrm{DCX}^{+}$cells and the associated cognitive deficits were not due to a higher death rate of newborn neurons. In addition, the increase in $\mathrm{DCX}^{+}$cells did not correlate with lesion size (Supplemental Figure 6 , A and B; $P>0.05$ vs. sham operated) or reductions in the number of postnatal generated neurons as a consequence of ischemic stroke (Supplemental Figure 7).

Enhancement of poststroke neurogenesis exacerbates hippocampal cognitive deficits after ischemia. All these data suggest that levels of neurogenesis and memory deficits after ischemic injury are directly related. To reinforce this correlation, we tested to determine whether interventions directed to further enhance SGZ neu- 
A

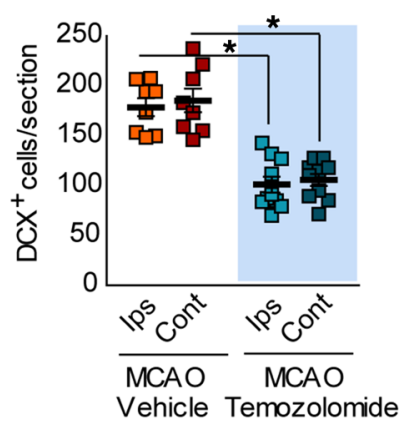

B
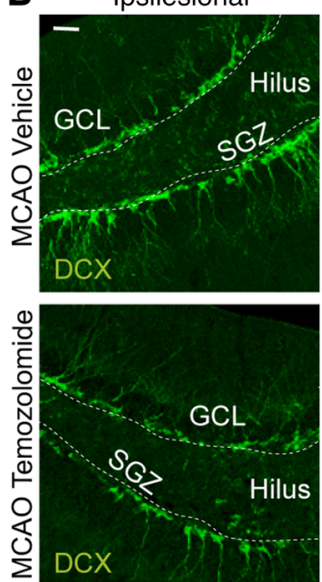

Contralesional

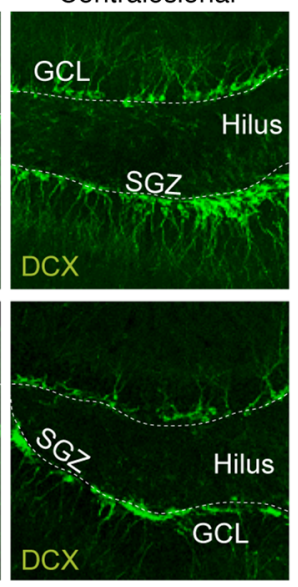

C

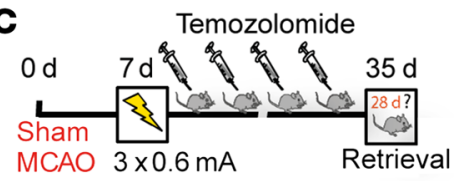

D

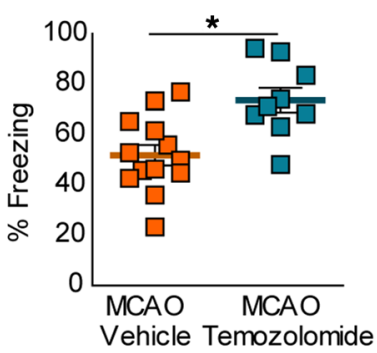

Figure 6. TMZ treatment after stroke mitigates hippocampus-dependent memory deficits. (A and B) Number of DCX+ cells in MCAO mice treated with vehicle or TMZ. Representative images of DCX+ cells (B, green) in MCAO mice treated with vehicle or TMZ. Scale bar: $30 \mu \mathrm{m}$. For A, 2-way ANOVA analysis showed a significant effect of TMZ in the number of $D C X^{+}$cells $\left(F_{(132)}=81.85 ; P<0.0001\right)$ in both ipsi- and contralesional sides (Bonferroni's post hoc: ${ }^{*} P<0.05$ vs. MCAO vehicle; MCAO vehicle, $n=8$; MCAO TMZ, $n=9$ ) at 35 days. (C) Experimental design for poststroke neurogenesis inhibition by TMZ. Seven days after ischemia, mice were subjected to CFC $(0.6 \mathrm{~mA} \times 3)$, and 24 hours later, they were treated i.p. with TMZ $(25 \mathrm{mg} / \mathrm{kg})$ for 4 weeks $(3$ days per week) and tested in the CFC at the end of the treatment. (D) Percentage of freezing response in vehicle- or TMZ-treated ischemic mice 35 days after CFC $\left({ }^{*} P<0.05\right.$ vs. MCAO vehicle; MCAO vehicle, $n=13$; MCAO TMZ, $\left.n=9\right)$. Data are represented as mean \pm SEM. Data were compared by using nonparametric 2-tailed Mann-Whitney $U$ tests (D) or nonparametric 2-way ANOVA followed by Bonferroni's post hoc testing (A).

rogenesis could exacerbate cognitive deficits in the MCAO group (Figure 4). First, 7 days after surgery, we enhanced neurogenesis in both sham-operated and MCAO mice by allowing them free access to a running wheel, while control animals remain sedentary, with a locked running wheel in their home cages. Twenty-eight days later, running efficiently increased the number of both proliferating Ki $67^{+}$cells (Figure 4, A and B; $P<0.05$ vs. MCAO sedentary) and $\mathrm{DCX}^{+}$cells (Figure $4, \mathrm{C}$ and $\mathrm{D} ; P<0.05$ vs. MCAO sedentary) in both sham-operated and MCAO groups compared with sedentary controls (Figure 4, A-D, and Supplemental Figure 8, A and B). To check whether overstimulated neurogenesis after stroke exacerbates cognitive impairment, we evaluated memory retention after CFC (Figure 4E). Consistent with our hypothesis, 28 days after conditioning, running further impaired the recall of remote contextual fear memories relative to that in the sedentary groups (Figure $4 \mathrm{E}$ and Supplemental Figure $8 \mathrm{C} ; P<0.05$ vs. sham operated or MCAO sedentary). In addition, MCAO impaired incidental context learning (Supplemental Figure 8D, $P<0.05$ vs. sham operated), an effect that was further increased by running (Supplemental Figure $8 \mathrm{D}, P<0.05$ vs. MCAO sedentary).

We next used the Barnes maze to determine whether a comparable effect was also found in spatial memory (Figure 4, F-I). Indeed, spatial memory deficits observed in the MCAO sedentary group were exacerbated in runner ischemic mice (Figure 4, $\mathrm{H}$ and I), which did not preferentially move toward the TQ (Figure $4 \mathrm{H}$, $P<0.05$ vs. MCAO sedentary) or the correct hole (Figure 4I, $P<$ 0.05 vs. MCAO sedentary), showing random behavior with no preference toward any region of the Barnes maze.

To reinforce our results, we next asked whether analogous memory deficits would be observed by using memantine (MEM) (Figure 4, J-L) as a pharmacological approach to increasing neurogenesis after stroke injury (28). Consistent with our previous results, MEM treatment increased poststroke SGZ neurogenesis, estimated as an increase in the number of $\mathrm{DCX}^{+}$cells (Figure 4, $\mathrm{J}$ and K, and Supplemental Figure 9A; $P<0.05$ vs. MCAO vehicle) while impairing remote memory recall, as demonstrated by a decreased freezing response (Figure $4 \mathrm{~L}$ and Supplemental Figure $9 \mathrm{~B} ; P<0.05$ vs. MCAO vehicle). All these data support the idea that enhanced hippocampal neurogenesis negatively interferes with the retrieval of memories and could therefore contribute to cognitive deficits after stroke.

Stroke-induced newborn neurons promote differential hippocampal circuitry remodeling. Memory retrieval may result from the reactivation of the same neuronal ensembles at the time of memory encoding $(29,30)$. Enhanced integration of newborn neurons into hippocampal circuits due to high neurogenesis levels could negatively regulate the ability to recall memories by replacing preexisting synapses and therefore remodeling hippocampal connections where memory is stored, a mechanism that has been demonstrated to mediate forgetting (9). This mechanism might thus explain the inverse correlation found between neurogenesis and hippocampal performance in our cortical ischemia model. However, in our scenario, an alternative and/or additional explanation might be the presence of pathological neurogenesis. Indeed, aberrant morphological alterations have been described for SGZ newborn neurons after MCAO $(18,19)$. To gain further insight into the mechanisms that contribute to cognitive impairment in our cortical stroke model, we characterized the distribution of the dendritic arborization along granular and molecular layers (ML) in immature neurons (DCX+' Supplemental Figure 10, A-D). Of note, differential patterns were found for ipsi- and contralesional DGs. While a clear increase in the percentage of dendritic arborization in the ML was observed for the contralesional hippocampus, no significant differences were detected for the ipsilesional side (Supplemental Figure 10, A and C; $P<0.05$ vs. sham operated and MCAO ipsilesional ML1, respectively). Inter- 
A

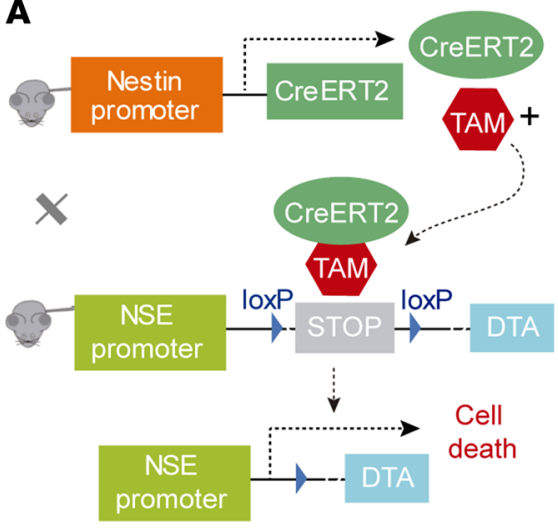

D
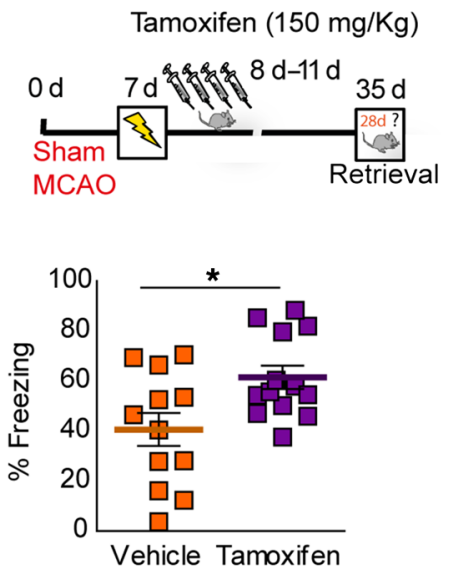

B

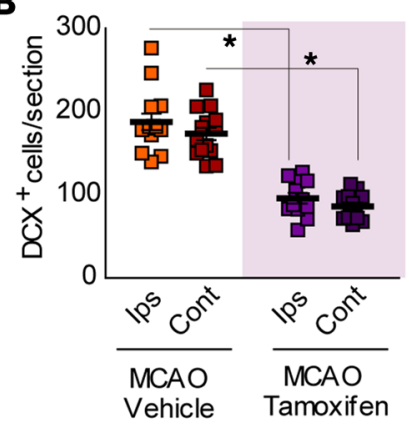

E

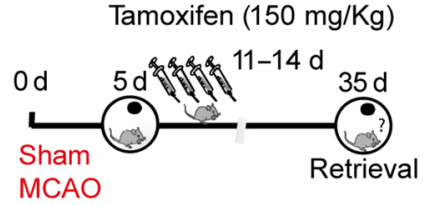

G

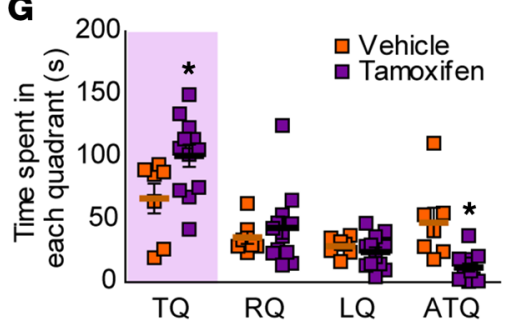

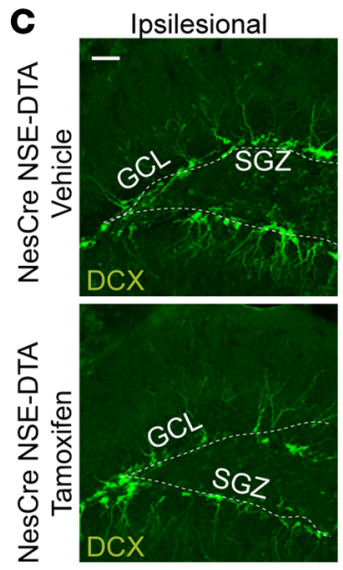

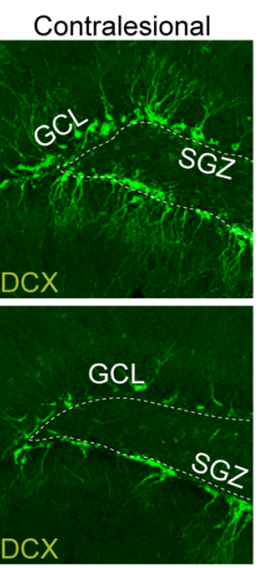

$\mathbf{F}$
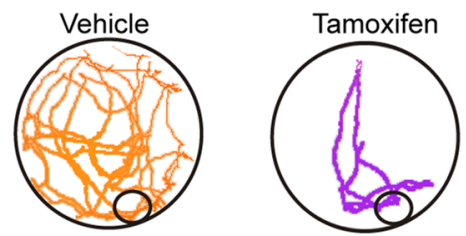

H

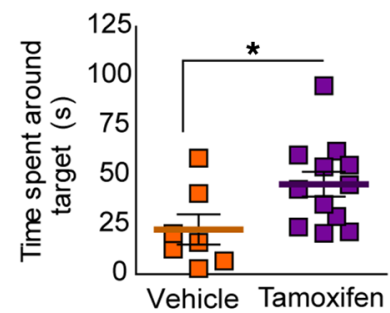

Figure 7. Conditional deletion of newborn neurons after stroke decreases cognitive deficits. (A) Genetic strategy used for conditional deletion of newborn neurons after stroke. (B) Number of DCX+ cells in MCAO Nestin-Cre ${ }^{\text {ERT2 } / N S E-D T A ~ m i c e ~ t r e a t e d ~ w i t h ~ v e h i c l e ~ o r ~ t a m o x i f e n . ~ T w o-w a y ~ A N O V A ~}$ showed a significant effect of tamoxifen in the number of $D C X^{+}$cells $\left(F_{(1,48)}=136.59 ; P<0.0001\right)$ in both ipsi- and contralesional sides (Bonferroni's post hoc: ${ }^{*} P<0.05$ vs. Nes-Cre ${ }^{\text {ERT2}} /$ NSE-DTA vehicle; vehicle, $n=14$; tamoxifen, $n=12$ ). (C) Representative images of DCX+ cells (green) in ischemic Nestin-Cre ${ }^{\text {ERT2 }}$ /NSE-DTA mice after vehicle or tamoxifen 35 days after surgery. Scale bar: $30 \mu \mathrm{m}$. (D) Percentage of freezing in vehicle- or tamoxifen-Nestin-Cre ${ }^{\text {ERT2}} /$ NSE-DTA treated mice 35 days after CFC ( ${ }^{*} P<0.05$ vs. Nestin-Cre ${ }^{\text {ERT2 }} /$ NSE-DTA vehicle; vehicle, $n=12$ and tamoxifen, $n=13$ ).

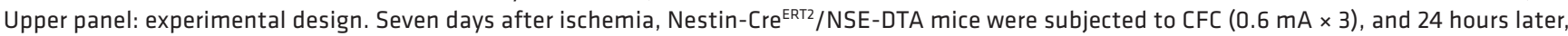
they were treated i.p. with vehicle or tamoxifen $(150 \mathrm{mg} / \mathrm{kg}$ ) over 4 consecutive days and tested in the CFC 35 days after surgery. (E-H) Experimental

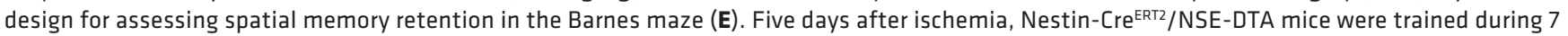
days ( 3 session/d) in the Barnes maze. After training, mice were treated with vehicle or tamoxifen and tested 28 days later. (F) Representative traces during the retention test. (G) Time spent in each quadrant of the Barnes maze platform. Two-way ANOVA demonstrated a significant interaction between quadrants and tamoxifen treatment in ischemic Nestin-Cre ${ }^{\text {ERT2 }} / \mathrm{NSE}-\mathrm{DTA}\left(\mathrm{F}_{(3.68)}=4.41 ; P=0.0068\right.$; Bonferroni's post hoc: ${ }^{*} P<0.05$ vs. Nestin-Cre ${ }^{\text {ERT2}} /$ NSE-DTA vehicle TQ). (H) Time spent around target hole during testing ( ${ }^{*} P<0.05$ vs. MCAO Nestin-Cre ${ }^{\text {ERT2 }} /$ NSE-DTA vehicle; vehicle, $n=7$; tamoxifen, $n=12)$. Data are represented as mean \pm SEM. Data were compared by using nonparametric 2-tailed Mann-Whitney $U$ tests $(\mathbf{D}$ and $\mathbf{H})$ and nonparametric 2-way ANOVA followed by Bonferroni's post hoc testing (B and $\mathbf{G}$ ).

estingly, differential results were also found in the number of immature large mossy fiber terminals $\left(\right.$ LMTs-DCX ${ }^{+}$) reaching the CA3 region. Whereas a reduction in the number of LMTs in CA3 was detected in the ipsilesional side, in contrast, an increased synaptic rearrangement was found in the contralesional CA3 (Supplemental Figure 10, B, D, and E; $P<0.05$ vs. sham operated and MCAO ipsilesional, respectively), further suggesting that differential remodeling processes were taking place in parallel at the ipsi- versus the contralesional hippocampus after ischemia.

To confirm these results, 14 days after surgery, we infused GFP-expressing retrovirus into the DG of sham-operated and ischemic mice in order to evaluate newborn neuron integration and possible circuit remodeling (Figure 5, A and B). Sholl analy- ses revealed a phenotype comparable to that observed for $\mathrm{DCX}^{+}$ arborization in contralesional DG $\mathrm{GFP}^{+}$neurons, corroborating an increase in dendritic branching in the distal segment (150-250 $\mu \mathrm{m}$ from the soma; Figure 5, C and D; $P<0.05$ vs. sham operated and MCAO ipsilesional, respectively) and in total dendritic length (Figure 5E; $P<0.05$ vs. sham operated and MCAO ipsilesional, respectively). Remarkably, an opposite phenotype was found in ipsilesional ischemic $\mathrm{GFP}^{+}$newborn neurons, with an increased degree of branching in the proximal domain $(0-50 \mu \mathrm{m}$ from the soma; Figure 5, C and D; $P<0.05$ vs. sham operated and MCAO contralesional, respectively) and a retraction of the distal domain in the ML. Accordingly, ipsilesional $\mathrm{GFP}^{+}$neurons also displayed a reduction in total dendritic length (Figure 5E). 
Mature granule newborn neurons generally display only 1 apical dendrite, which remains almost not ramified until it reaches the ML, where it begins to extend its branches and establishes synapses with afferents of the perforant pathway from the entorhinal cortex (EC). Therefore, apical dendrite growing seems to be a critical factor for the correct integration of newborn neurons $(31,32)$. Our data also demonstrate a differential pattern of apical dendrite growth in $\mathrm{GFP}^{+}$neurons of the ipsi- versus the contralesional ischemic DG. Contralesional ischemic $\mathrm{GFP}^{+}$neurons presented a longer apical dendrite compared with both ipsilesional and sham-operated $\mathrm{GFP}^{+}$neurons (Figure $5 \mathrm{~F}$ and Supplemental Figure 11A). In contrast, ipsilesional $\mathrm{GFP}^{+}$neurons showed a dramatic reduction in apical dendrite length compared with the other groups (Figure 5F; $P<0.05$ vs. sham operated and MCAO contralesional, respectively). Interestingly, when we classified $\mathrm{GFP}^{+}$ neurons in each group according to apical dendrite length intervals (Figure 5G and Supplemental Figure 11A), our data demonstrate that apical dendrite growth alteration due to ischemic stroke differentially affected approximately $30 \%$ of the newborn neuron population in ipsi- (predominant aberrant phenotype $<10 \mu \mathrm{m}$ ) and contralesional sides (predominant aberrant phenotype 40-70 $\mu \mathrm{m}$ ), compared with the $\mathrm{GFP}^{+}$neurons observed in sham-operated mice (predominant phenotype 10-40 $\mu \mathrm{m}$ ). We also analyzed to determine whether stroke affected spine density of $\mathrm{GFP}^{+}$newborn neurons as an index of synaptic integration. Of note, despite their altered dendrite arborization, no differences were observed in the density of dendritic spines of $\mathrm{GFP}^{+}$newborn granule cells either in the ipsi- or in the contralesional side when compared with the sham-operated group (Supplemental Figure $11, \mathrm{~B}$ and $\mathrm{C}$ ), suggesting that these newborn neurons are integrated into hippocampal circuits receiving inputs from the EC.

As previously demonstrated for poststroke neurogenesis, aberrant features of newborn neurons might also be transient after stroke. For assessing this issue, in a different set of experiments, the GFP-expressing retrovirus was delivered 35 days after stroke (Supplemental Figure 12A), when poststroke neurogenesis had already returned to physiological levels. Interestingly, although the aberrant features of newborn neurons previously detected in Sholl analysis or in mean apical dendrite length were not observed at this time point (Supplemental Figure 12, B and C), 30.5\% of the ipsilesional newborn population still displayed apical dendrite growth alterations (aberrant phenotype $<10 \mu \mathrm{m}$; Supplemental Figure 12, D and E), an effect that might lead to an incorrect integration of axonal projections coming from EC.

All these results indicate that ischemic stroke promotes an increase in neurogenesis that gives rise to the generation of differential populations of newborn neurons with altered morphological features depending on their ipsi- or contralesional location, an effect that remains, at a lower intensity, when the levels of neurogenesis become normalized. The integration of these abnormal neurons could promote aberrant hippocampal circuitry rearrangements and, therefore, contribute to hippocampal cognitive deficits observed after ischemia.

Poststroke memory impairment is reduced by downregulation of ischemia-induced aberrant neurogenesis. Our data support that high levels of poststroke neurogenesis initiate a differential remodeling of hippocampal circuits that contributes to cognitive deficits after stroke. Therefore, we predicted that decreasing aberrant poststroke neurogenesis toward physiological levels might ameliorate ischemia-induced hippocampal deficits. For this, we used both pharmacological and genetic strategies. First, we suppressed poststroke neurogenesis by treating mice with temozolomide (TMZ), a DNA-alkylating agent (Figure 6 and Supplemental Figure 13). TMZ treatment caused a reduction of neurogenesis in MCAO mice, as reflected by reduced numbers of proliferating Ki67 ${ }^{+}$cells and immature neurons expressing DCX (Figure 6, A and B; $P<0.05$ vs. MCAO vehicle, and Supplemental Figure 13). Importantly, and confirming our hypothesis, TMZ treatment improved memory retrieval in parallel to a reduction in neurogenesis, as shown by an increased freezing response in mice evaluated 28 days later (Figure 6, $\mathrm{C}$ and $\mathrm{D} ; \mathrm{P}<0.05$ vs. MCAO vehicle).

To reinforce our results, we genetically ablated aberrant hippocampus-generated neurons after ischemia by using Nes-

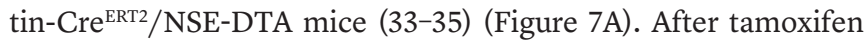
administration, a STOP region in the diphtheria toxin fragment A (DTA) cassette gene was deleted in nestin ${ }^{+}$neural stem cells (NSCs). These cells die by apoptosis at the beginning of their neuronal differentiation, due to the DTA expression under the neuron-specific enolase 2 (NSE, also known as Eno2) promoter gene. Genetic ablation of newly formed neurons after stroke led to a decrease in $\mathrm{DCX}^{+}$cells (Figure 7, B and C; $P<0.05$ vs. Nestin-Cre ${ }^{\text {ERT2 }} /$ NSE-DTA vehicle, and Supplemental Figure 14, A and B) in parallel to a better performance in both contextual fear (Fig-

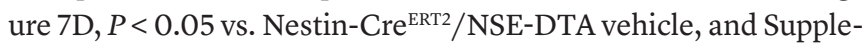
mental Figure 14C) and the Barnes maze test (Figure 7, E-H, $P<$

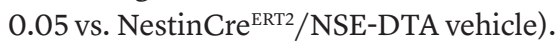

Together, our data demonstrate that increased levels of poststroke neurogenesis contribute to cognitive hippocampal deficits observed following injury, very likely by producing aberrant remodeling of hippocampal circuits.

\section{Discussion}

Ischemic stroke is currently considered one of the most disabling illnesses (1). Most of the studies in this field have been focused on strategies to limit the expansion of the initial damage as well as the motor effects (2). However, clinical-pathological studies have revealed stroke as a major contributor to cognitive impairment in patients (reviewed in refs. $5,36,37$ ). Due to the heterogeneity of the mechanisms underlying cognitive deficits and the lack of effective animal models to recapitulate this condition, the pathophysiological knowledge and the development of treatments for poststroke cognitive impairment remain an urgent therapeutic challenge. In the present study, we have investigated the mechanisms underlying long-term memory deficits driven by cortical ischemic stroke. Through a series of studies, we show that cortical ischemia in mice promotes memory impairment due, at least partly, to a pathological hippocampal circuitry remodeling prompted by the poststroke hippocampal neurogenic response.

First, in our cortical stroke model, we observed a long- but not short-term impairment in both episodic and spatial memories after ischemic stroke, supporting the idea that, after this type of acute damage, there is a progressive and persistent decline of cognitive function that resembles that observed in stroke patients $(2,3,5,10,36)$. The impairment was independent of whether the 
memory was formed before, early after, or even long term after stroke, at least for the times and conditioning protocols tested in this study. The risk of developing poststroke cognitive impairment has been associated with the extent of lesion size and its strategic location (37). In order to study the mechanisms associated with a specific location, we took advantage of the low degree of variability and the cortical location of our stroke model, which allowed us to discard brain structural alterations located outside of the cortical ischemic lesion that could also participate in the observed cognitive impairment.

In the adult mammal brain, the hippocampus is one of the main regions implicated in cognitive function. Importantly, although our MCAO model neither directly affects the overall hippocampal anatomy nor causes a secondary long-term atrophy, we have confirmed the implication of this structure by different approaches. First, hippocampus inactivation by TTX infusion completely abolished the ability of retrieval in both MCAO and sham-operated groups, indicating that a correct activation of this structure is critical to bringing the memory back after our stroke model. Second, stroke hindered the proper activation of the hippocampus to recall old memories, determined as a reduction in the number of hippocampal c-Fos ${ }^{+}$cells after long-term retrieval. All these results point to the hippocampus as the main contributor for poststroke memory impairment in our cortical ischemia model.

Adult hippocampal neurogenesis occurs in SGZ of the DG, which is considered a source of new neurons that persists in the adult hippocampus of most mammals, including humans (8, 38, 39). Thus, newborn neurons are continually being generated, providing new potential substrates for the incoming information (4043). Importantly, numerous examples have been provided for the role of physiological and pathological neurogenesis in hippocampus-dependent learning and memory processes $(10,11,44)$. Using a long-term temporal study, we describe a longitudinal profile of the neurogenic response in both ipsi- and contralesional SGZ during the chronic phase of stroke. Specifically, we have observed an increased number of proliferative cells 2 weeks after stroke onset and of immature newborn neurons (neuroblasts) that remained enhanced 1 month after the ischemic injury. This neurogenic response could account for the augmentation of the GCL volume, since the majority of stroke-induced $\mathrm{DCX}^{+}$cells were finally fully integrated into the hippocampal circuits at the time studied. Interestingly, an inverse correlation was found between neurogenesis levels and memory recall, supporting the idea that higher levels of SGZ neurogenesis are a modulating factor of cognitive performance that may contribute to impaired memory retrieval after stroke. This idea was reinforced by the results obtained after enhancing poststroke neurogenesis by physical activity or by a pharmacological approach using $\operatorname{MEM}(25,28)$, showing a more severe cognitive decline in contextual and spatial memory after ischemia.

Whereas the addition of newborn cells in the DG provides new potential sources for storing incoming information, the integration of newborn neurons may also promote a remodeling of circuits where memory is stored, weakening existing memories, a phenomenon known as "forgetting" $(9,45)$. This body of evidence implies that, due to the net increase in newborn neurons after stroke, remodeling of hippocampal circuits could drive the forgetting of previously acquired information. The vast majority of cells generated in the SGZ differentiate into dentate granule cells, extending (a) their apical dendrite toward the ML where it extensively ramifies, and (b) their axons, called large mossy fiber terminals, toward targets in the CA3 region, forming input and output synaptic connections $(17,40,46)$. However, after stroke, newborn neurons display different morphological alterations that might hinder their proper integration into the hippocampal circuits (19). Importantly, our results show a different remodeling process occurring simultaneously at each side of the hippocampus after ischemic stroke, demonstrated by distinctive morphological alterations observed in newborn neurons at the ipsi- and contralesional hippocampi. Indeed, by analyzing immature and mature newborn neurons, our results are the first demonstration, to our knowledge, of a different phenotype of newborn neurons at the ipsilesional when compared with contralesional hippocampus that seems to affect both synaptic inputs and outputs, beyond the physiological "forgetting" induced by enhanced neurogenesis. On the one hand, one important difference between phenotypes is the different pattern of dendritic arborization probably driven by a differential apical dendrite growth. Whereas ipsilesional ischemic neurons show an increased proximal dendritic branch density, contralesional neurons display an elongation of apical dendrite and, consequently, an increase in the distal dendrite branch. This altered dendritic branching could have important consequences for incoming information from the EC, since inputs in the inner and outer MLs carry different information (47), and thus could have profound functional implications at the network level. On the other hand, our data suggest that stroke also promotes a differential bilateral remodeling in the connectivity between DG and CA3. Each new cell in the DG projects a mossy fiber that reaches the CA3 region within approximately 2 weeks, contacting with 11-15 pyramidal cells (40, 42, 48). Interestingly, in CA3, we report a hyper- or a hypointegration pattern of immature newborn neurons at the contra- and ipsilesional hemispheres, respectively. These data could imply an increased synaptic rearrangement in the contralesional CA3, which could coexist or replace the previous DG-CA3 synapses. Although we have not confirmed this result in full mature neurons, newly generated neurons transiently (2-6 weeks of the cell age) display enhanced synaptic plasticity $(49,50)$, suggesting that these immature new neurons may also transiently have the ability to deprive preexisting synapses and, therefore, contribute to deficits observed in our model.

The transient nature of poststroke-induced neurogenesis suggests that the production of abnormal newborn granule neurons could also be temporary. Indeed, we have observed that aberrant features of newborn neurons decrease at later times after stroke, in agreement with a return of neurogenesis to basal levels; however, our data show that the generation of aberrant newborn neurons, although to a substantially lower extent, persists at later times, suggesting that the factors that induce morphological remodeling, albeit likely decreasing over time, are still present at later time points. This is to be added to the important fraction of aberrant neurons generated during the stroke-induced neurogenic burst that will likely remain abnormally integrated into the hippocampal circuits. They are likely to contribute to the persistent cognitive deficits found at the latest time points studied. 
As noted above, when testing hippocampus-dependent memory in a retrograde fashion, increasing neurogenesis after memory acquisition induces forgetting due to the interference caused by the integration of newborn neurons within the preexisting hippocampal circuits (9). However, the generation of new mature granule neurons in the hippocampus has been traditionally thought to be an endogenous mechanism for cognitive repair, in agreement with a positive effect of neurogenesis on anterograde memories by which new neurons synaptically integrate into hippocampal circuits offering a substrate for acquisition of new memories (learning). In the context of cerebral ischemia, a substantial amount of evidence supports a positive role of neurogenesis in stroke models. Of note, in most models used, the injury directly affects the hippocampus, as after global ischemia $(51,52)$ or after models of focal ischemia with hippocampal affectation $(15,53,54)$. In similar settings, physical exercise, an intervention known to increase neurogenesis, has been shown to promote neurocognitive recovery in rodents (reviewed in refs. 8, 55). However, in these studies, basal affectation of hippocampus caused by a direct ischemic injury is likely to explain the positive effect of neurogenesis. It should be noted that some studies have reported positive effects of neurogenesis on cognitive function in stroke models without hippocampal affectation. For instance, hypoxic postconditioning stimuli increased neurogenesis in parallel to a better cognitive performance (56); however, in this case, abolition of neurogenesis did not affect cognitive function in MCAO-exposed rats. In this line, genetic abolition of neurogenesis has been described as worsening cognitive function after stroke (17); nevertheless, neurogenesis ablation was performed prior to the MCAO procedure, which may be the cause for those mice being likely to display a basal cognitive deficit on which the effects of neurogenesis, as in the damaged hippocampus, are likely to be beneficial. Therefore, the positive effect of neurogenesis on anterograde memories after stroke, including when physical exercise interventions are tested, is demonstrated when the hippocampus shows some degree of affectation, a situation that does not correspond with our experimental setting, in which the hippocampus remains intact after the MCAO. In contrast, and similarly to our results, other studies have observed the detrimental effect of stroke in the morphological features and correct integration of newborn granule cells, in parallel to impaired spatial memory $(18,19)$.

Apart from physical exercise interventions, pharmacological tools such as MEM and fluoxetine have been described as being useful in increasing neurogenesis $(28,57)$. By this mechanism, MEM has been shown to exert a negative effect on retrograde memories (forgetting) $(9,58)$. This might seem to be in conflict with the current clinical use of this drug, which is the treatment of moderate to severe Alzheimer's disease (AD). However, the well-known action of MEM as a noncompetitive, low- to mediumaffinity antagonist of brain NMDA glutamate receptors is likely the major mechanism that accounts for its beneficial effect in AD.

Our data suggest that an aberrant hippocampal circuitry remodeling due to stroke-induced neurogenic response could explain, at least partly, poststroke cognitive impairment. To confirm our hypothesis, we abolished stroke-induced neurogenesis using pharmacological (TMZ) or genetic approaches

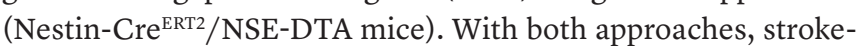

induced increase in neurogenesis was ablated, as shown by a return to basal levels in numbers of both proliferating cells and immature newborn neurons at the DG. Remarkably, a major result of our present work is that, after these strategies, MCAO mice displayed an improvement in contextual and spatial memory recall performance, supporting the important role of strokeinduced neurogenesis in cognitive impairment. Considering this as a pathological response, we are the first, to our knowledge, to propose the inhibition of aberrant hippocampal poststroke neurogenesis with the aim of avoiding the onset of long-term memory deficits after stroke.

Several mechanisms could account for poststroke aberrant neurogenesis. On the one hand, mediators of postischemic excitotoxicity or cortical spreading depression, such as $\mathrm{K}^{+}$and glutamate elevations, could be involved, as both have been implicated in an enhanced proliferation of immature cells while at the same time directing toxicity in more mature cell types $(59,60)$. Another influence to be considered is the inflammatory response elicited by the injured brain, whereby astrocytes and microglia activation could lead to the secretion of a variety of growth factors and immune modulators able to affect progenitor proliferation and survival $(61,62)$. In addition, injury-induced circulating factors might directly affect neural progenitors that are in physical contact with the vasculature (63).

Overall, our results identify hippocampal circuitry remodeling after stroke-induced neurogenesis response as a process that promotes the loss of hippocampus-dependent memories. We show that stroke-induced neurogenesis could be considered as a therapeutic target for the treatment of the cognitive decline shown by stroke patients, opening new lines of research in the development of treatments for this devastating condition.

\section{Methods}

\section{Animals}

Experiments were performed in 8- to 10-week-old male C57BL/6 mice obtained from The Jackson Laboratory. NSE-DTA mice (NSE-Stop-DTA mice) were donated by S. Itohara and Y. Kobayashi (RIKEN Center for Brain Science, Saitama Japan; refs. 34, 35) and were maintained through homozygous breeding pairs. Nestin-Cre ${ }^{\text {ERT2 }} /$ NSE-DTA mice were generated by crossing NSE-DTA mice with Nestin-Cre ${ }^{\text {ERT2 }}$ mice (33) and then maintained through homozygous breeding pairs on a C57BL/6 background. In these transgenic mice (Nestin-CreERT2/NSE-DTA), tamoxifen-inducible Cre is expressed by NSCs under the nestin promoter and the loxP-STOP-loxP-IRES-DTA gene cassette is knocked into the NSE gene. After tamoxifen treatment, Cre recombinase deletes the STOP sequence in the NSC pool. Throughout maturation, the NSE promoter becomes active, inducing the expression of DTA, resulting in cellular programmed death. Thus, the generation of fully mature newborn granule neurons is dramatically decreased in these mice. The following primers were used for genotyping: Nestin-CreERT2 mice, 5'-TTCCGCTGGGTCACTGTCGCCGCTAC-3' and 5'-TAATCGCGAACATCTTCAGGTTCTGC-3'; NSE-DTA mice, 5'-AATTCTTAATTAAGGCGCGCCGG-3', 5'-GTCAGAATTGAGGAAGAGCTGGGG-3', and 5'-CACTGAGGATTCTTCTGTGG-3'.

Animals were kept in a room under controlled temperature with a 12-hour dark/12-hour light cycle and fed with standard food and water ad libitum. 
For all experiments, $n$ represents biologically independent samples, except for animals shown in Figure 1B, which were used for the correlations shown in Figure 3, Supplemental Figure 2, A-C, and Supplemental Figure 4B, and animals in sham-operated and MCAO control groups, which were used for testing the effect of running and TMZ on both neurogenesis levels and CFC memory, as shown in Figures 4 and 6 and Supplemental Figures 8 and 13.

\section{MCAO in mice}

Mice were subjected to permanent focal cerebral ischemia through the distal occlusion of middle cerebral artery (MCA), as previously described (64). Briefly, mice were anesthetized with isoflurane $1.5 \%-2 \%$ in a mixture of $80 \%$ air/ $20 \%$ oxygen, and body temperature was maintained at physiological levels with a heating pad during the surgical procedure and anesthesia recovery. Mice were subjected to permanent focal cerebral ischemia (MCAO) through the distal occlusion of MCA by ligature of the trunk just before its bifurcation between the frontal and parietal branches with a 9-0 suture, in combination with the occlusion of the ipsilesional common carotid artery. Mice in which the MCA was exposed but not occluded served as sham-operated controls. Following surgery, individual animals were returned to their home cages with free access to water and food. All the experiments were performed and quantified in a randomized fashion by investigators blinded to treatment groups.

\section{Infarct volume measurement}

Infarct size was determined by magnetic resonance imaging 24 to 48 hours after MCAO using a Biospec BMT 47/40 (Bruker). Infarct volume was calculated using ImageJ software (NIH) from the T2-weighted images as described (65). In addition, infarct size was determined by Nissl staining as described (64).

\section{Determination of hippocampus and DG volume}

The volume of the hippocampus and the DG were estimated stereologically by applying the Cavalieri method, performed with Stereo Investigator software (MicroBrightField), for each series of Nisslstained sections. Volumes were estimated by using a 1 -in- 5 systematic random series of $30 \mu \mathrm{m}$ Nissl-stained sections using a 100 and $20 \mu \mathrm{m}^{2}$ point-counting grid, respectively.

\section{Treatments}

Running. Mice in running groups (3 mice/cage) were given voluntary access to 2 running wheels placed in their home cages. Access to wheels started 7 (CFC) or 12 days (Barnes maze) after ischemia and 24 hours after training. Sedentary controls were similarly housed, but running wheels were locked during all the procedures.

Tamoxifen. Nestin-Cre ${ }^{\text {ERT2 }} /$ NSE-DTA mice were injected i.p. daily with tamoxifen $(150 \mathrm{mg} / \mathrm{kg})$ in sunflower oil for 5 consecutive days starting 7 days after ischemia and 24 hours after training.

$B r d U$. For experiments aimed at studying the number of newborn granule neurons 65 days after MCAO, BrdU was dissolved in PBS and injected in 2 rounds of treatment at 1-week intervals, with each round consisting of 1 injection (100 mg/kg, i.p.) per day for 5 consecutive days and starting 10 days after ischemia. For postnatal labeling, BrdU was administered for 5 consecutive days, 1 injection $(100 \mathrm{mg} / \mathrm{kg}$, i.p. $)$ per day, and starting when mice were 1 month old.
MEM. Mice were administered MEM (0.9\% saline, $25 \mathrm{mg} / \mathrm{kg}$, i.p.) once per week over 4 weeks as previously described (58). MEM administration started 7 days after ischemia and 24 hours after training.

TMZ. Mice were given 4 rounds of treatment at 1-week intervals, with each round consisting of 1 injection of TMZ (0.9\% in saline containing 10\% DMSO, $25 \mathrm{mg} / \mathrm{kg}$, i.p.) per day for 4 consecutive days (66). TMZ administration started 7 days after ischemia and 24 hours after training.

TTX. Hippocampus activity was transiently inactivated by infusion of TTX (a sodium channel blocker) (67). Three hours before the retrieval session, mice received an infusion of $0.5 \mu \mathrm{l}$ of TTX (20 $\mu \mathrm{M}$, Wako) at a rate of $0.2 \mu \mathrm{l} / \mathrm{min}$ into the hippocampus ( $-2 \mathrm{~mm} \mathrm{AP}$, $-1.7 \mathrm{~mm}$ ML relative to bregma, and $-2 \mathrm{~mm}$ DV from skull) with a $5 \mu \mathrm{l}$ 32s-gauge Hamilton syringe.

Retrovirus. New neurons were labeled using a murine Moloney leukemia virus-based retroviral vector (CAG-GFP, Addgene; ref. 40). Concentrated viral solutions were prepared by transfection of retroviral vectors into Gryphon Eco cells, followed by ultracentrifugation of viral supernatant and concentrated virus solution by ultraspeed centrifugation to a concentration of $1 \times 10^{8}$ to $1 \times 10^{9}$ viral particles $/ \mathrm{ml}$. Mice were anesthetized with isoflurane and placed in a stereotaxic frame.

Retrovirus $(2 \mu \mathrm{l})$ was infused at a rate of $0.2 \mu \mathrm{l} / \mathrm{min}$ into the DG (-2 $\mathrm{mm}$ AP, $-1.4 \mathrm{~mm}$ ML relative to bregma, and $-2.4 \mathrm{~mm}$ DV from skull), with a $5 \mu \mathrm{l} 32 \mathrm{~s}$-gauge Hamilton syringe. Retroviral injections were performed 14 or 35 days after surgery in both sham-operated and MCAO groups, and morphological analysis was performed 28 days later.

\section{Behavioral testing}

CFC. CFC occurred in test chambers $(31 \mathrm{~cm} \times 24 \mathrm{~cm} \times 21 \mathrm{~cm})$ with shock-grid floors (bars $3.2 \mathrm{~mm}$ in diameter spaced $7.9 \mathrm{~mm}$ apart). The front, top, and back of the chamber were clear acrylic, and the sides were modular aluminium. CFC was performed 7 days after surgery in both sham-operated and MCAO groups, except for a set of experiments in which conditioning was performed 48 hours before surgery or 30 days after surgery. During conditioning, mice were placed in the chamber and, after 150 seconds of familiarization, they received 3 foot shocks ( $0.6 \mathrm{~mA}, 2$ seconds duration, 1 minute apart). Mice were removed from the chamber 1 minute after the last shock. In an additional set of experiments, a weak $(0.4 \mathrm{~mA} \times 2$, 2 seconds duration, 1 minute apart) or a strong $(0.8 \mathrm{~mA} \times 5,2$ seconds duration, 1 minute apart) conditioning protocol was also used. During the retrieval test, mice were placed in the chamber for 5 minutes. Behavior was recorded by overhead cameras and freezing (i.e., absence of movement except for breathing) quantified with a chronometer by blinded observers. In addition, activity, inactivity, and grooming were also measured.

Incidental context learning. Incidental context learning in mice occurred in the same chambers as described above. During the context preexposure session (performed 7 days after surgery), mice were placed in the chambers for 10 minutes. During the immediate shock session, mice were returned to the chambers, a single foot shock was immediately delivered ( $\leq 1$ seconds after entry, $1 \mathrm{~mA}, 2$ seconds duration), and mice were removed from the chambers after 1 minute. During the retrieval session ( 24 hours later), mice were placed in the chambers for 5 minutes.

Barnes maze. A white circular platform $(100 \mathrm{~cm}$ diameter, $70 \mathrm{~cm}$ above the floor) contained 20 holes equally spaced around its perimeter. Under one of the holes there was a black Plexiglas escape box (17 
$\times 13 \times 7 \mathrm{~cm}$ ) filled with paper bedding. The location of this escape hole was always in the same place for all mice. For avoiding navigation based on olfactory or proximal cues within the maze, the platform was rotated before each trial, and the spatial location of the escape hole remained in a fixed location with respect to the distal room cues. During habituation, mice were allowed 5 minutes to freely explore the maze, with no escape box present. During training, mice were given 3 trials per day for 7 consecutive days. For each trial, mice were released in the center of the maze and allowed 10 minutes to enter the escape box, where they remained for 30 seconds. If a mouse failed to find the escape box, it was guided by the experimenter. During the retrieval test, the escape box was removed from the maze, and mice were allowed to search for 3 minutes. Behavior was recorded by overhead cameras and tracked using automated EthoWatcher software (UFSC). Time spent around each hole and around each quadrant was measured.

\section{Histology}

Tissue preparation. Mice were perfused transcardially with phosphate buffer $(0.1 \mathrm{M})$ followed by $4 \%$ paraformaldehyde (PFA). Brains were post-fixed in PFA and transferred to $30 \%$ sucrose. Coronal sections $(30 \mu \mathrm{m})$ were cut using a microtome (Leica SM2000R) and stored in cryoprotective solution.

Nissl staining. Brain tissue sections were placed in $0.5 \%$ cresyl violet in distilled water for 10 minutes at room temperature. Briefly, sections were rinsed in $75 \%, 90 \%$, and $100 \%$ ethanol twice for 5 minutes before being dehydrated in xylene 3 times for 5 minutes. Sections were mounted with Permount.

Immunohistochemistry. Immunofluorescence was performed on free-floating sections that were incubated overnight at $4^{\circ} \mathrm{C}$ with the following primary antibodies: goat anti-calbindin (1:500, Santa Cruz Biotechnology Inc., catalog sc-7691), mouse anti-NeuN (1:500, Merck, catalog MAB377), goat anti-DCX (1:500, Santa Cruz Biotechnology Inc., catalog sc-8066), rabbit anti-DCX (1:500, Abcam, catalog ab18723), rabbit anti-c-Fos (1:1000, Abcam, catalog 7963, and Merck, catalog PC05L), rabbit anti-mouse Ki67 (1:500, Abcam, catalog ab137876), chicken anti-GFP (1:700, Thermo Fisher Scientific, catalog A10262), and goat anti-Znt3 (1:3000, Santa Cruz Biotechnology Inc., catalog sc-27508). For BrdU staining, free-floating sections were pretreated with $2 \mathrm{~N} \mathrm{HCl}$ for 30 minutes at $37^{\circ} \mathrm{C}$ and then incubated overnight at $4^{\circ} \mathrm{C}$ with rat anti-5-bromo-2'-deoxyuridine (BrdU; 1:500, AbD Serotec, catalog 0BT0030S). For cleaved caspase-3 staining, sections were pretreated with $0.01 \mathrm{M}$ citrate buffer $(\mathrm{pH} 6.0)$ in a $97^{\circ} \mathrm{C}$ steamer for 15 minutes and allowed to cool to room temperature for $20 \mathrm{~min}$ utes and then incubated overnight with rabbit anti-cleaved caspase-3 (1:100, Cell Signalling Technology, catalog 9661S). The secondary antibodies used were donkey Alexa Fluor 488 anti-goat (1:500, Thermo Fisher Scientific, catalog A11055), donkey Cy3 anti-mouse (1:500, Merck; catalog AP192), goat Alexa Fluor 488 anti-chicken (1:500, Thermo Fisher Scientific, catalog A11039), and donkey Cy3 antirabbit (Merck, catalog AP182C). Controls performed in parallel without primary antibodies showed very low levels of nonspecific staining.

Image acquisition, processing and quantification. Acquisitions were performed with a laser-scanning confocal imaging system (Zeiss LSM710), and image analysis was carried out with ZEN2009 (Zeiss) software. Image quantification was performed with ImageJ. The number of $\mathrm{Ki}^{+}$and DCX cells were counted in confocal $Z$-stack images, for which $4-8$ serial sections $(30 \mu \mathrm{m})$ per animal spaced $300 \mu \mathrm{m}$ apart (from bregma $-1.46 \mathrm{~mm}$ to bregma $-2.30 \mathrm{~mm}$ ) were used. $\mathrm{Ki}^{+} 7^{+}$and $\mathrm{DCX}^{+}$cells were counted manually in frames of $212.55 \mu \mathrm{m} \times 212.55 \mu \mathrm{m}(1024 \times 1024)$. Data are expressed as the number of cells per section.

For assessing the number of newborn neurons in the hippocampus $\left(\mathrm{Brdu}^{+} / \mathrm{NeuN}^{+}\right.$or $\mathrm{BrdU}^{+} /$calbindin ${ }^{+}$cells) and the number of c-Fos cells, 10 hippocampal sections, sampled every fifth section (from bregma $-1.46 \mathrm{~mm}$ to bregma $-2.30 \mathrm{~mm}$ ) were used. The total number of $\mathrm{BrdU}^{+}$cells in the DG were calculated by multiplying the count in each section by 10 and then totalling the values. The images were taken at $\times 63$, and colocalization of $\mathrm{NeuN}$ or calbindin ${ }^{+}$with $\mathrm{BrdU}^{+}$was confirmed by orthogonal projection of $Z$-stack files. c-Fos ${ }^{+}$cells were counted manually in different hippocampal regions (CA1, CA3, and DG), and total cell numbers were calculated by multiplying the count in each section by 10 and then totalling the values of all regions as the sum of CA1, CA3 and DG c-Fos ${ }^{+}$cells. c-Fos ${ }^{+}$cell numbers in the conditioned groups (both sham operated and MCAO) were expressed as a percentage relative to the untrained/unconditioned group (mice that were exposed to the context, but did not receive any foot shocks).

For morphometric analysis, 2 series of $50 \mu \mathrm{m}$ coronal sections from each animal were used for immunohistochemical detection of GFP-labeled neurons. Confocal $\times 40$ stacks of images were obtained, and $\mathrm{z}$ projections were used to trace neurons using the NeuronJ plugin for ImageJ software. Sholl's analysis was then performed in order to determine dendritic complexity (determination of total dendritic length, apical dendrite length, and degree of dendritic arbor branching) using the plugin Sholl analysis for ImageJ (68). This analysis consists of placing a central point on the cellular soma and tracing concentric circles with a $10 \mu \mathrm{m}$ distance interval between them. Each intersection between dendritic branches and circles was represented (68). For spinal analysis, images of GFP-labeled dendritic processes at the outer ML were acquired at $0.5 \mu \mathrm{m}$ intervals with a plain apochromatic $\times 63$ oil lens (numerical aperture [NA], 1.4) and a digital zoom of 3. The $l s m$ image files were subjected to 2 iterations of deconvolution with the AutoDeblur program (AutoQuant). The length of each dendritic segment was measured, and the number of spines was counted manually. Spine density was calculated by dividing the total number of spines by the length of the dendritic segment. For quantification, 10-15 dendritic segments from each animal in an experimental group were used. Confocal imaging and data quantification were performed by researchers blinded to the experimental conditions.

\section{Statistics}

Results are expressed as the mean \pm SEM for the indicated number of experiments, and statistical analyses were performed using Prism5 software (GraphPad Software). Comparisons between 2 groups were performed using nonparametric Mann-Whitney $U$ test, while those involving more than 2 groups were performed using nonparametric 1-way ANOVA or 2-way ANOVA followed by Bonferroni's post hoc test. Correlation analyses were performed using Spearman's correlation. Differences were considered statistically significant at $P<0.05$.

\section{Study approval}

All experimental protocols performed were approved by the Animal Welfare Committee of the Universidad Complutense and the Regional Government of Madrid (following EU directives 86/609/CEE and 2003/65/CE and Spanish directives RD53/2013). 


\section{Author contributions}

MIC, JDP, and MAM designed the research studies. MIC, JDP, APR, VDL, IBF, and AGC conducted the experiments and/or acquired the data. JD and PWF provided valuable reagents. MIC, JDP, JMGS, JD, PWF, IL, and MAM contributed to the analysis and/or interpretation of the results. MIC, JDP, IL, and MAM wrote the manuscript, which all authors reviewed and approved.

\section{Acknowledgments}

This work was supported by grants from the Spanish Ministry of Economy and Competitiveness (MINECO) (SAF2015-68632-R to MAM), the Instituto de Salud Carlos III (ISCIII) (FIS PI17/01601 to IL), and ISCIII cofinanced by the Fondo Europeo de Desarrol- lo Regional (FEDER) "Una manera de hacer Europa" RETICS (RD12/0014/0003 to IL), and the Canadian Institutes of Health Research (FDN143227to PWF).

Address correspondence to: María A. Moro or María Isabel Cuartero, Unidad de Investigación Neurovascular, Departamento de Farmacología y Toxicología, Facultad de Medicina, Universidad Complutense, Pza. Ramón y Cajal s/n, 28040 Madrid, Spain. Phone: 34.913941452. Email: neurona@med.ucm.es (M.A. Moro) or maribel_cd@hotmail.com (M.I.Cuartero).

MIC's present address is: Centro de Biología Molecular Severo Ochoa, CSIC, Spain.
1. Benjamin EJ, et al. Heart disease and stroke statistics-2017 update: a report from the American Heart Association. Circulation. 2017;135(10):e146-e603.

2. Mijajlović MD, et al. Post-stroke dementia - a comprehensive review. BMC Med. 2017;15(1):11.

3. Levine DA, et al. Trajectory of cognitive decline after incident stroke. JAMA. 2015;314(1):41-51.

4. Brainin M, et al. Post-stroke cognitive decline: an update and perspectives for clinical research. Eur J Neurol. 2015;22(2):229-238, e13.

5. Pendlebury ST, Rothwell PM. Prevalence, incidence, and factors associated with prestroke and post-stroke dementia: a systematic review and meta-analysis. Lancet Neurol. 2009;8(11):1006-1018.

6. Tatemichi TK, Desmond DW, Stern Y, Paik M, Sano M, Bagiella E. Cognitive impairment after stroke: frequency, patterns, and relationship to functional abilities. J Neurol Neurosurg Psychiatry. 1994;57(2):202-207.

7. Hu GC, Chen YM. Post-stroke dementia: epidemiology, mechanisms and management. Int J Gerontol. 2017;11(4):210-214.

8. Zhao C, Deng W, Gage FH. Mechanisms and functional implications of adult neurogenesis. Cell. 2008;132(4):645-660.

9. Akers KG, et al. Hippocampal neurogenesis regulates forgetting during adulthood and infancy. Science. 2014;344(6184):598-602.

10. Mu Y, Gage FH. Adult hippocampal neurogenesis and its role in Alzheimer's disease. Mol Neurodegener. 2011;6:85.

11. Cho KO, et al. Aberrant hippocampal neurogenesis contributes to epilepsy and associated cognitive decline. Nat Commun. 2015;6:6606.

12. Schreglmann SR, et al. The temporal expression pattern of alpha-synuclein modulates olfactory neurogenesis in transgenic mice. PLoS One. 2015;10(5):e0126261.

13. Aimone JB, Li Y, Lee SW, Clemenson GD, Deng $\mathrm{W}$, Gage FH. Regulation and function of adult neurogenesis: from genes to cognition. Physiol Rev. 2014;94(4):991-1026.

14. Lichtenwalner RJ, Parent JM. Adult neurogenesis and the ischemic forebrain. J Cereb Blood Flow Metab. 2006;26(1):1-20.

15. Kernie SG, Parent JM. Forebrain neurogenesis after focal Ischemic and traumatic brain injury. Neurobiol Dis. 2010;37(2):267-274.

16. Raber J, et al. Irradiation attenuates neurogenesis and exacerbates ischemia-induced deficits. Ann Neurol. 2004;55(3):381-389.

17. Sun C, et al. Conditional ablation of neuroprogenitor cells in adult mice impedes recovery of poststroke cognitive function and reduces synaptic connectivity in the perforant pathway. J Neurosci. 2013;33(44):17314-17325.

18. Niv F, Keiner S, Krishna -, Witte OW, Lie DC, Redecker C. Aberrant neurogenesis after stroke: a retroviral cell labeling study. Stroke. 2012;43(9):2468-2475.

19. Woitke F, et al. Adult hippocampal neurogenesis poststroke: More new granule cells but aberrant morphology and impaired spatial memory. PLoS One. 2017;12(9):e0183463.

20. Epp JR, Silva Mera R, Köhler S, Josselyn SA, Frankland PW. Neurogenesis-mediated forgetting minimizes proactive interference. $\mathrm{Nat} \mathrm{Com-}$ mun. 2016;7:10838.

21. Al-Qazzaz NK, Ali SH, Ahmad SA, Islam S, Mohamad K. Cognitive impairment and memory dysfunction after a stroke diagnosis: a post-stroke memory assessment. Neuropsychiatr Dis Treat. 2014;10:1677-1691.

22. Anagnostaras SG, Gale GD, Fanselow MS. Hippocampus and contextual fear conditioning: recent controversies and advances. Hippocampus. 2001;11(1):8-17.

23. Altman J, Das GD. Autoradiographic and histological evidence of postnatal hippocampal neurogenesis in rats. J Comp Neurol. 1965;124(3):319-335.

24. Kempermann G, Kuhn HG, Gage FH. More hippocampal neurons in adult mice living in an enriched environment. Nature. 1997;386(6624):493-495

25. van Praag H, Kempermann G, Gage FH. Running increases cell proliferation and neurogenesis in the adult mouse dentate gyrus. Nat Neurosci. 1999;2(3):266-270.

26. Arvidsson A, Kokaia Z, Lindvall O. N-methyl$\mathrm{D}$-aspartate receptor-mediated increase of neurogenesis in adult rat dentate gyrus following stroke. Eur J Neurosci. 2001;14(1):10-18.

27. Kluska MM, Witte OW, Bolz J, Redecker C. Neurogenesis in the adult dentate gyrus after cortical infarcts: effects of infarct location, N-methyl-Daspartate receptor blockade and anti-inflammatory treatment. Neuroscience. 2005;135(3):723-735.

28. Maekawa M, Namba T, Suzuki E, Yuasa S, Kohsaka S, Uchino S. NMDA receptor antagonist memantine promotes cell proliferation and pro- duction of mature granule neurons in the adult hippocampus. Neurosci Res. 2009;63(4):259-266.

29. Girardeau G, Benchenane K, Wiener SI, Buzsáki G, Zugaro MB. Selective suppression of hippocampal ripples impairs spatial memory. Nat Neurosci. 2009;12(10):1222-1223.

30. Dupret D, O’Neill J, Pleydell-Bouverie B, Csicsvari J. The reorganization and reactivation of hippocampal maps predict spatial memory performance. Nat Neurosci. 2010;13(8):995-1002.

31. Shapiro LA, Upadhyaya P, Ribak CE. Spatiotemporal profile of dendritic outgrowth from newly born granule cells in the adult rat dentate gyrus. Brain Res. 2007;1149:30-37.

32. Llorens-Martín M, Jurado-Arjona J, Avila J, Hernández F. Novel connection between newborn granule neurons and the hippocampal CA2 field. Exp Neurol. 2015;263:285-292.

33. Imayoshi I, Ohtsuka T, Metzger D, Chambon P, Kageyama R. Temporal regulation of Cre recombinase activity in neural stem cells. Genesis. 2006;44(5):233-238.

34. Kobayakawa K, et al. Innate versus learned odour processing in the mouse olfactory bulb. Nature. 2007;450(7169):503-508.

35. Kobayashi Y, et al. Genetic dissection of medial habenula-interpeduncular nucleus pathway function in mice. Front Behav Neurosci. 2013;7:17.

36. Gorelick PB, et al. Vascular contributions to cognitive impairment and dementia: a statement for healthcare professionals from the American Heart Association/American Stroke Association. Stroke. 2011;42(9):2672-2713.

37. Iadecola C. The pathobiology of vascular dementia. Neuron. 2013;80(4):844-866.

38. Ming GL, Song H. Adult neurogenesis in the mammalian central nervous system. Annu Rev Neurosci. 2005;28:223-250.

39. Spalding KL, et al. Dynamics of hippocampal neurogenesis in adult humans. Cell. 2013;153(6):1219-1227.

40. Zhao C, Teng EM, Summers RG, Ming GL, Gage FH. Distinct morphological stages of dentate granule neuron maturation in the adult mouse hippocampus. J Neurosci. 2006;26(1):3-11.

41. Toni N, et al. Synapse formation on neurons born in the adult hippocampus. Nat Neurosci. 2007;10(6):727-734.

42. Toni $\mathrm{N}$, et al. Neurons born in the adult dentate gyrus form functional synapses with target cells. Nat Neurosci. 2008;11(8):901-907. 
43. Gu Y, et al. Optical controlling reveals timedependent roles for adult-born dentate granule cells. Nat Neurosci. 2012;15(12):1700-1706.

44. Winner B, Winkler J. Adult neurogenesis in neurodegenerative diseases. Cold Spring Harb Perspect Biol. 2015;7(4):a021287.

45. Frankland PW, Köhler S, Josselyn SA. Hippocampal neurogenesis and forgetting. Trends Neurosci. 2013;36(9):497-503.

46. Restivo L, Niibori Y, Mercaldo V, Josselyn SA, Frankland PW. Development of adult-generated cell connectivity with excitatory and inhibitory cell populations in the hippocampus. J Neurosci. 2015;35(29):10600-10612.

47. Lisman JE. Role of the dual entorhinal inputs to hippocampus: a hypothesis based on cue/ action (non-self/self) couplets. Prog Brain Res. 2007;163:615-625.

48. Acsády L, Kamondi A, Sík A, Freund T, Buzsáki G. GABAergic cells are the major postsynaptic targets of mossy fibers in the rat hippocampus. J Neurosci. 1998;18(9):3386-3403.

49. Schmidt-Hieber C, Jonas P, Bischofberger J. Enhanced synaptic plasticity in newly generated granule cells of the adult hippocampus. Nature. 2004;429(6988):184-187.

50. Ge S, Yang CH, Hsu KS, Ming GL, Song H. A critical period for enhanced synaptic plasticity in newly generated neurons of the adult brain. Neuron. 2007;54(4):559-566.

51. Nakatomi H, et al. Regeneration of hippocampal pyramidal neurons after ischemic brain injury by recruitment of endogenous neural progenitors. Cell. 2002;110(4):429-441.
52. Bendel O, Bueters T, von Euler M, Ove Ogren S, Sandin J, von Euler G. Reappearance of hippocampal CA1 neurons after ischemia is associated with recovery of learning and memory. JCereb Blood Flow Metab. 2005;25(12):1586-1595.

53. Luo CX, et al. Voluntary exercise-induced neurogenesis in the postischemic dentate gyrus is associated with spatial memory recovery from stroke. J Neurosci Res. 2007;85(8):1637-1646.

54. Li WL, et al. Chronic fluoxetine treatment improves ischemia-induced spatial cognitive deficits through increasing hippocampal neurogenesis after stroke. J Neurosci Res. 2009;87(1):112-122.

55. van Praag H. Neurogenesis and exercise: past and future directions. Neuromolecular Med. 2008;10(2):128-140.

56. Tsai YW, Yang YR, Wang PS, Wang RY. Intermittent hypoxia after transient focal ischemia induces hippocampal neurogenesis and c-Fos expression and reverses spatial memory deficits in rats. PLoS ONE. 2011;6(8):e24001.

57. Malberg JE, Eisch AJ, Nestler EJ, Duman RS. Chronic antidepressant treatment increases neurogenesis in adult rat hippocampus. J Neurosci. 2000;20(24):9104-9110.

58. Ishikawa R, Fukushima H, Frankland PW, Kida S. Hippocampal neurogenesis enhancers promote forgetting of remote fear memory after hippocampal reactivation by retrieval. Elife. 2016;5:e17464.

59. Shi J, Miles DK, Orr BA, Massa SM, Kernie SG. Injury-induced neurogenesis in Bax-deficient mice: evidence for regulation by voltagegated potassium channels. Eur J Neurosci. 2007;25(12):3499-3512.
60. Mattson MP. Glutamate and neurotrophic factors in neuronal plasticity and disease. Ann N Y Acad Sci. 2008;1144:97-112.

61. Myer DJ, Gurkoff GG, Lee SM, Hovda DA, Sofroniew MV. Essential protective roles of reactive astrocytes in traumatic brain injury. Brain. 2006;129(Pt 10):2761-2772.

62. Bessis A, Béchade C, Bernard D, Roumier A. Microglial control of neuronal death and synaptic properties. Glia. 2007;55(3):233-238.

63. Mignone JL, Kukekov V, Chiang AS, Steindler D, Enikolopov G. Neural stem and progenitor cells in nestin-GFP transgenic mice. JComp Neurol. 2004;469(3):311-324.

64. Ballesteros I, Cuartero MI, Moraga A, de la Parra J, Lizasoain I, Moro MA. Stereological and flow cytometry characterization of leukocyte subpopulations in models of transient or permanent cerebral ischemia. J Vis Exp. 2014;(94):52031.

65. Cuartero MI, et al. L-kynurenine/aryl hydrocarbon receptor pathway mediates brain damage after experimental stroke. Circulation. 2014;130(23):2040-2051.

66. Garthe A, Behr J, Kempermann G. Adult-generated hippocampal neurons allow the flexible use of spatially precise learning strategies. PLoS One. 2009;4(5):e5464.

67. Kitamura T, et al. Adult neurogenesis modulates the hippocampus-dependent period of associative fear memory. Cell. 2009;139(4):814-827.

68. Ferreira T, Ou Y, Li S, Giniger E, van Meyel DJ. Dendrite architecture organized by transcriptional control of the F-actin nucleator Spire. Development. 2014;141(3):650-660. 